\title{
Clinical applications of optical coherence tomography in the posterior pole: the 20I I José Manuel Espino Lecture - Part II
}

This article was published in the following Dove Press journal:

Clinical Ophthalmology

5 November 2013

Number of times this article has been viewed

\section{J Fernando Arevalo ${ }^{1,2}$ \\ Andres $\mathrm{F}$ Lasave ${ }^{3}$ \\ Juan D Arias ${ }^{3}$ \\ Martin A Serrano ${ }^{3}$ \\ Fernando A Arevalo ${ }^{3}$}

'Retina Division, Wilmer Eye Institute, Johns Hopkins University School of Medicine, Baltimore, MD, USA; ${ }^{2}$ Vitreoretinal Division, King Khaled Eye Specialist Hospital, Riyadh, Kingdom of Saudi Arabia; ${ }^{3}$ Retina and Vitreous Service, Clinica Oftalmologica Centro Caracas, Caracas, Venezuela
Correspondence: J Fernando Arevalo Vitreoretinal Division, The King Khaled Eye Specialist Hospital, Al-Oruba Street, PO Box 7I9I, Riyadh II462,

Kingdom of Saudi Arabia

Tel +966 I 482 I234 ext 3860

Fax +966 I 482 I 234 ext 3727

Email arevalojf@jhmi.edu

\begin{abstract}
Optical coherence tomography (OCT) is a high-resolution, cross-sectional imaging technique that allows detailed assessment of retinal thickness and morphologic evaluation of the retinal layers. This technology has developed quickly over the past two decades. OCT imaging has rapidly been integrated into routine ophthalmic clinical practice and trials. It has complemented fluorescein angiography in many instances, especially in the diagnosis and management of retinal disorders, including diabetic macular edema and age-related macular degeneration. With OCT, the exact localization of pathologic features can be visualized in segmentation maps of the retina, and this has allowed OCT to be used to evaluate specific features that may serve as predictive factors in the prognosis and follow up of these pathologies. Therefore, it has become an important clinical and research tool for the diagnosis, follow up, treatment, and assessment of new treatment modalities for all diseases that affect the posterior pole of the eye.
\end{abstract}

Keywords: AMD, DME, retinal diseases, spectral domain

\section{Introduction}

Optical coherence tomography (OCT) is a medical diagnostic imaging technology that can perform micrometer-resolution, cross-sectional or tomographic imaging in biologic tissues. In recent years, OCT has been established as a useful tool in the diagnosis and clinical evaluation of macular diseases because it allows detailed assessment of retinal thickness and morphologic evaluation of the neurosensory retinal layers. In addition, it complements fluorescein angiography (FA) in the evaluation of most common retinal disorders, including macular edema and age-related macular degeneration.

In patients with diabetes and diabetic retinopathy, some studies have shown that single measurements of the central foveal thickness, using OCT, correlate with visual acuity and are a successful means of monitoring macular thickening before and after therapy. ${ }^{1-5}$ However, other studies have reported conflicting results. ${ }^{6-7}$ Changes in diabetic macular edema can be accurately and prospectively measured with OCT, in both clinical trials and clinical practice. In neovascular age-related macular degeneration (AMD), OCT is able to measure retinal thickness accurately and reproducibly, and it is effective in assessing the response to treatment for choroidal neovascularization and is increasingly being used to guide treatment decisions. On the other hand, the improved image quality and faster image-acquisition speed of spectral domain (SD)-OCT has turned more attention toward patients with non-neovascular AMD, because different types of drusen are being identified; thus, studies are now in progress to detect the early markers for progression to late stage non-neovascular or neovascular AMD. 
OCT has become an important clinical and research tool for the diagnosis, follow up, treatment, and assessment of new therapy modalities for any macular disease.

In Part I of this review we discussed the basic principles, interpretation, and clinical applications of OCT in the vitreoretinal interface disorders of the posterior pole, including macular hole, lamellar macular hole, and macular pseudohole, epiretinal membrane, vitreomacular traction syndrome, and the relationship between vitreomacular traction and AMD.

In this second part of our review we discuss the role of this technology in a variety of retinal pathologies that affect the posterior pole (the retina between the optic disc and the macula) including AMD, diabetic retinopathy, and various macular diseases. The topics discussed in this review do not pretend to be all inclusive and do not include some important diseases that can affect the posterior pole, such as intraocular tumors, uveitis, and inherited retinal diseases.

\section{Age-related macular degeneration}

Despite recent progress, AMD remains the leading cause of irreversible blindness among individuals older than 60 years, in industrialized countries. ${ }^{8}$ Cigarette smoking, genetic factors, aging, ischemia, and environmental factors are considered the main important etiological factors of AMD. ${ }^{8}$ Other additional factors influencing the development of exudative AMD include changes at the vitreomacular interface, which have been confirmed by OCT in several studies. ${ }^{9,10}$ Early non-neovascular AMD is characterized by the accumulation of macular drusen and changes in pigmentation, whereas late AMD progresses to geographic atrophy (GA), neovascularization, and severe loss of central vision.

\section{Diagnostic methods}

Fundus imaging provides an important contribution to the early and precise detection and diagnosis of AMD. Color fundus photographs and autofluorescence images document anatomical landmarks to monitor the progression of signs of AMD, with its exudative and hemorrhagic complications. However, only angiographic methods can directly visualize choroidal neovascularization (CNV); FA demonstrates classic CNV and indocyanine green angiography can better demonstrate occult CNV.

Currently, the determination of whether CNV is classic or occult in type is no longer necessary for the initiation of treatment because the management of AMD therapy with antiangiogenic agents now largely involves determining whether or not $\mathrm{CNV}$ exists, regardless of type.
OCT continues to evolve, with image resolution improving from 5 to 10 microns, using the time-domain techniques, to less than 5 microns, using the SD techniques. ${ }^{11}$ Most importantly, OCT is able to demonstrate the exudative component related to the abnormal permeability of CNV. SD-OCT techniques can also directly analyze the impact of $\mathrm{CNV}$ on the outer retinal layers and evaluate alterations of the photoreceptors and inner and outer segments.

OCT is critical for nearly every aspect of AMD management. Although OCT can be useful in the diagnosis of non-neovascular AMD, the true value is apparent in the management of neovascular AMD because OCT can help to establish treatment and retreatment guidelines and help to monitor treatment failure or success. This technology can identify indirect signs related to abnormal exudation, such as intraretinal fluid or increased retinal thickness, and it can be used to characterize drusen, GA, subretinal neovascularization and CNV, neurosensory retinal detachment, and pigment epithelial detachments (PED), which are associated with AMD. ${ }^{12}$ Therefore, OCT has become an essential part of the examination in routine follow up for AMD, to guide clinical practice and to analyze the response to treatment.

Previously, when considering retreatment in AMD, FA was commonly used as an indicator of CNV activity; however, several reports have indicated poor agreement in the interpretation of FA in AMD, especially after intravitreal treatment. ${ }^{13,14}$ The treatment of CNV with inhibitors of vascular endothelial growth factor (VEGF) requires monthly evaluation of the activity of the lesion to support the decision for retreatment, and OCT can be very useful. ${ }^{15}$

Recent studies have validated a good agreement in the interpretation of Stratus (Carl Zeiss Meditec, Jena, Germany) time-domain OCT (TD-OCT) images between observers and also good correlation between FA and TD-OCT, in terms of retinal thickness, subretinal fluid thickness, and CNV thickness. ${ }^{13,16}$ According to repeated studies, OCT might partially replace FA in the follow up of exudative AMD. ${ }^{17,18}$

\section{Tomographic characteristics in age-related macular degeneration \\ Geographic atrophy}

GA is a significant cause of both moderate and severe central visual loss and is bilateral in most patients with advanced AMD. It represents the loss of photoreceptors, retinal pigment epithelium (RPE), and choriocapillaris within the macula. GA is highly distinctive on OCT because the overlying retina is thinned and the hypopigmented RPE causes increased penetration of the optical probe beam into the deeper choroid, significantly enhancing the reflections from this layer. 
The intense back shadowing in the zones of atrophy, extending well into the choroid, is the defining sign of atrophy. Another sign, on OCT, is the thinning of the neurosensory retina over the zones of atrophy, which is particularly serious when it involves the central zone. In addition, there are no exudative signs. Thinning and loss of the RPE are clearly visualized, but maintenance of the straight line representing Bruch's membrane (BM) is an important sign (Figures 1 and 2). In the most severe forms, the outer nuclear layer is no longer

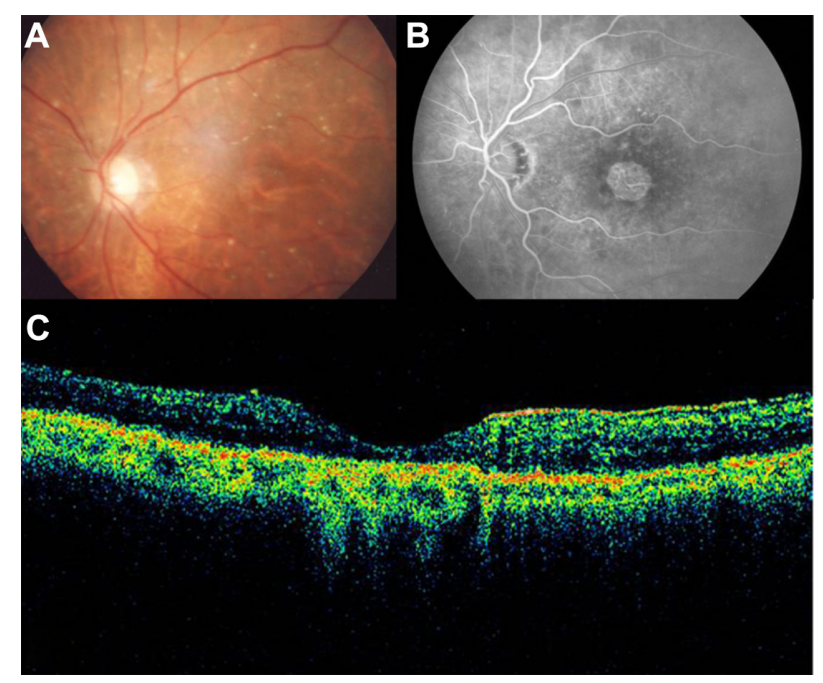

Figure I (A) Color fundus photograph of geographic atrophy, secondary to agerelated macular degeneration, showed a rounded, depigmented area with welldefined margins. (B) Fluorescein angiography demonstrated hyperfluorescence due to a window defect. (C) The Stratus optical coherence tomography image showed backscatter in the zones of atrophy, with marked hyperreflectivity and retinal thinning in the entire zone of atrophy.

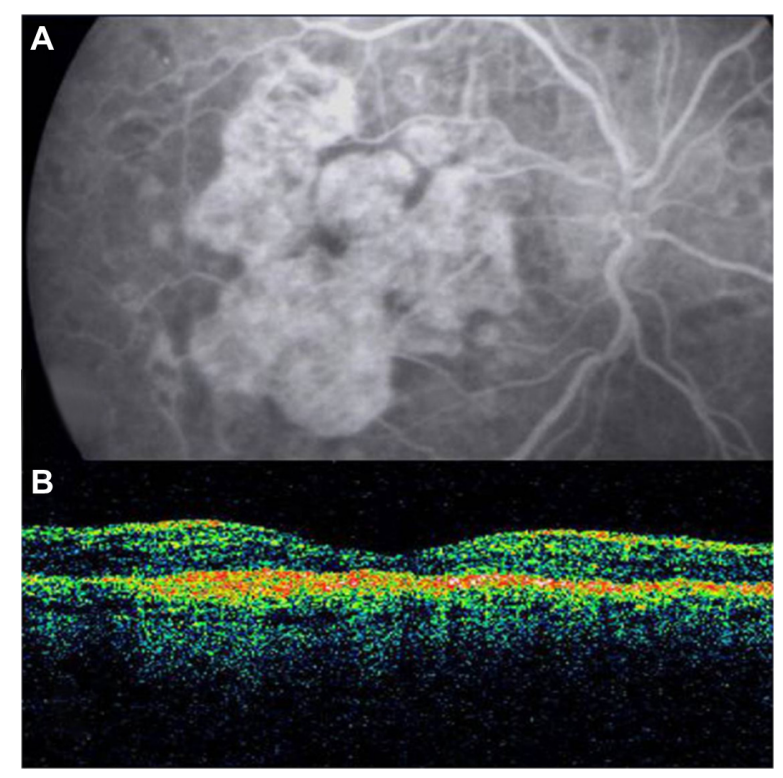

Figure 2 Geographic atrophy. (A) Fluorescein angiography demonstrated hyperfluorescence due to a window defect and central sparing. (B) Stratus optical coherence tomography image showing a significant loss of the outer nuclear layer, photoreceptors, and the retinal pigment epithelium, leading to significant hyperreflectivity and backscatter within the choroid. visible in the zones of atrophy. RPE atrophy is irreversible and causes the death of almost all the photoreceptors in the atrophic area, resulting in a corresponding scotoma.

Due to the current absence of an effective treatment, dry AMD has a poor prognosis, despite the hopes raised by a recognized clinical trial (Age-Related Eye Disease Study [AREDS]) that demonstrated the benefits of antioxidants, vitamin A, and vitamin C supplements. Dietary antioxidant supplementation reduced the risk of severe vision loss in intermediate forms of dry AMD and severe AMD, but it produced no conclusive effect on the primary prevention of early AMD. ${ }^{19,20}$

\section{Drusen analysis}

Despite great advances in fundus image analysis in the past 20 years, with the development of various manual and automated tools for drusen analysis, fundus photos have been the mainstay of drusen characterization until the introduction of OCT. These tools are capable of providing qualitative and quantitative analysis of drusen changes.

Gass noted that an increase in the drusen area was associated with a higher risk of progression to advanced AMD. ${ }^{21}$ Several published studies have also shown that a greater drusen diameter and area may be associated with a significant risk of progression to advanced AMD within 5 years. ${ }^{22-24}$ Small hard drusen are considered an early sign of AMD. ${ }^{25}$ Large numbers of hard drusen have been shown to progress to GA. ${ }^{26}$ In the Blue Mountains Eye study, the eyes with large soft drusen were six times more likely to develop advanced AMD compared with the eyes without such drusen. ${ }^{27-29}$ Elsewhere, it was shown that approximately $25 \%$ of the large drusen present at baseline faded over 10 years of follow up, in the absence of apparent clinical progression of AMD. ${ }^{30,31}$

Soft drusen are observed as focal elevations in the external highly reflective band (RPE/choriocapillaris complex), consistent with the accumulation of amorphous material within or beneath BM. SD-OCT has been able to consistently identify the morphological parameters of the drusen ultrastructure, which can be used to better understand the natural history of drusen (Figure 3 ) and to monitor drusen change in patients undergoing treatment.

\section{Pigment epithelial detachment}

A retinal pigment epithelial detachment (PED) is formed by the separation of the RPE from BM due to the presence of sub-RPE fluid, blood, fibrovascular membrane, or drusenoid material. ${ }^{32,33}$ PED is an important predictor of vision loss in patients with AMD. Around half of patients with a newly diagnosed PED will experience an average visual loss of $>3$ 
lines over 1 year of follow up. ${ }^{34}$ Four main types of PEDs are found in patients with AMD: drusenoid, serous, fibrovascular, and hemorrhagic. PEDs are a common presentation of neovascular AMD, seen in up to $62 \%$ of eyes with evidence of advanced AMD, by FA. ${ }^{9}$

A drusenoid PED is a fairly well-circumscribed, shallow, and often multiple elevation of the reflective band corresponding to the RPE/choriocapillaris complex, formed by one or more large drusen or as a result of the slow coalescence of soft drusen $^{33,35,36}$ (Figures 3 and 4). It has been distinguished from the other types of PED by its relatively better prognosis. ${ }^{37}$ However, the natural history usually follows a progression to persistent drusenoid PED, GA, and neovascularization. ${ }^{38,39}$

Drusenoid PED has a particular evolution and potential risks for severe loss of visual acuity, and it should be considered as a subgroup of AMD. Recently, anti-VEGF therapy ${ }^{40-42}$ has shown variable anatomical and visual outcomes in patients with symptomatic drusenoid PED without $\mathrm{CNV}$ in AMD (Figures 5 and 6).

Serous PED (SPED) are sharply demarcated, smooth, dome-shaped elevations of the RPE (Figure 7). ${ }^{43}$ By OCT, a SPED is identified as a smooth domed elevation of the RPE, with a characteristic sharp angle of PED over an optically empty space bound inferiorly by a visible BM (Figure 8). ${ }^{35}$

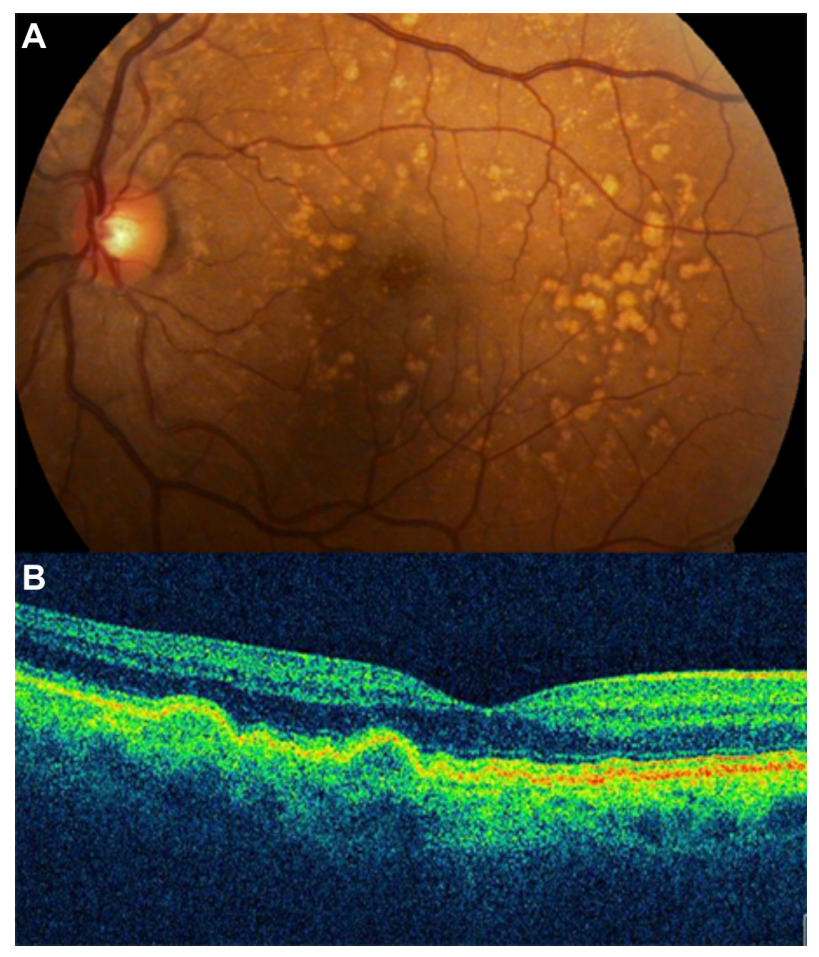

Figure 3 (A) Color photograph. (B) Cirrus ${ }^{\mathrm{TM}}$ optical coherence tomography image. Notes: In (B), a drusenoid pigment epithelial detachment is a fairly well circumscribed, shallow, and often multiple elevation of the reflective band corresponding to the retinal pigment epithelium/choriocapillaris complex, formed by one or more large drusen or as a result of the slow coalescence of soft drusen.

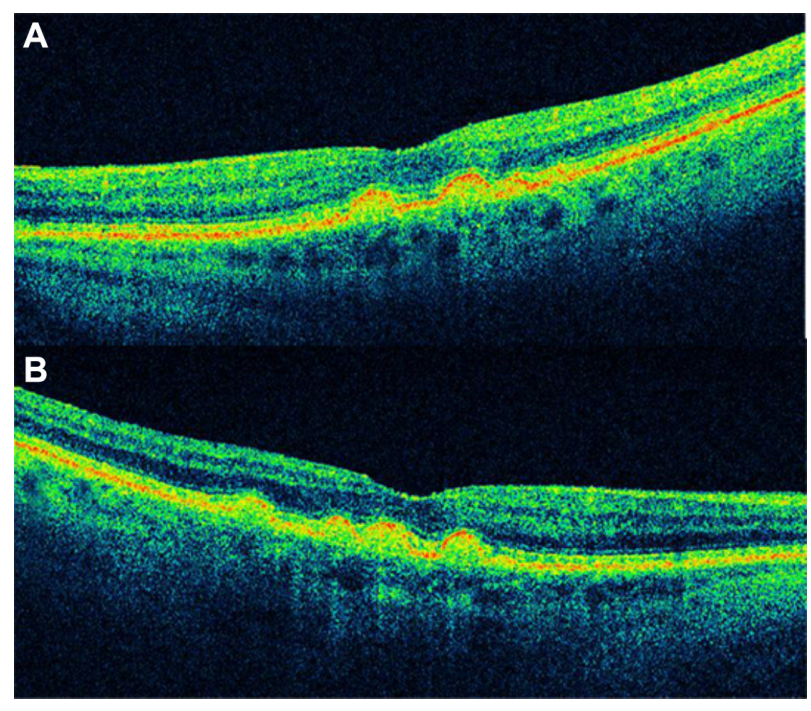

Figure 4 (A and B) Bilateral drusenoid pigment epithelial detachment is observed on Cirrus ${ }^{\mathrm{TM}}$ optical coherence tomography.

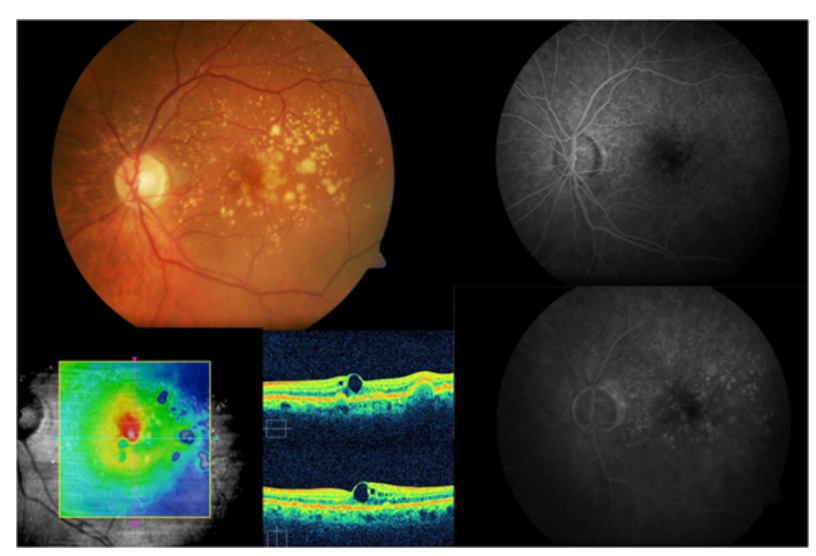

Figure 5 Cirrus $^{\mathrm{TM}}$ optical coherence tomography scan.

Notes: Baseline visit: a 67-year-old male complained of blurred vision on his left eye. Retinography demonstrated soft confluent drusen within the macular area (top left). The best-corrected visual acuity was $20 / 50$. Cirrus ${ }^{\mathrm{TM}}$ optical coherence tomography revealed a drusenoid pigment epithelial detachment, and microcystic hyporeflective spaces within the neurosensory retina and an elevated retinal map (bottom left). Fluorescein angiography did not show any associated choroidal neovascularization (top and bottom right). Reprinted with permission from GallegoPinazo R, Marina A, Suelves-Cogollos, et al. Intravitreal ranibizumab for symptomatic drusenoid pigment epithelial detachment without choroidal neovascularization in age-related macular degeneration. Clin Ophthalmol. 201 I;5:16I-165. Copyright 20I I Dove Medical Press. ${ }^{42}$

Classically, the edges of the dome are sharply demarcated (Figure 9). An area of active neovascularization associated with a SPED may lead to an associated smaller cuff of subretinal and intraretinal fluid that buttresses the SPED, a characteristic finding on OCT cross section (Figures 10 and 11). With antiVEGF therapy, this area of subretinal fluid will often improve despite persistence of the associated PED.

Similarly, the combination of a PED with accompanying subretinal fluid and intraretinal fluid may be a sign of $\mathrm{CNV}$ or retinal angiomatous proliferation (Figure 12). In contrast to $\mathrm{CNV}$-associated SPEDs, RAP-associated SPEDs 


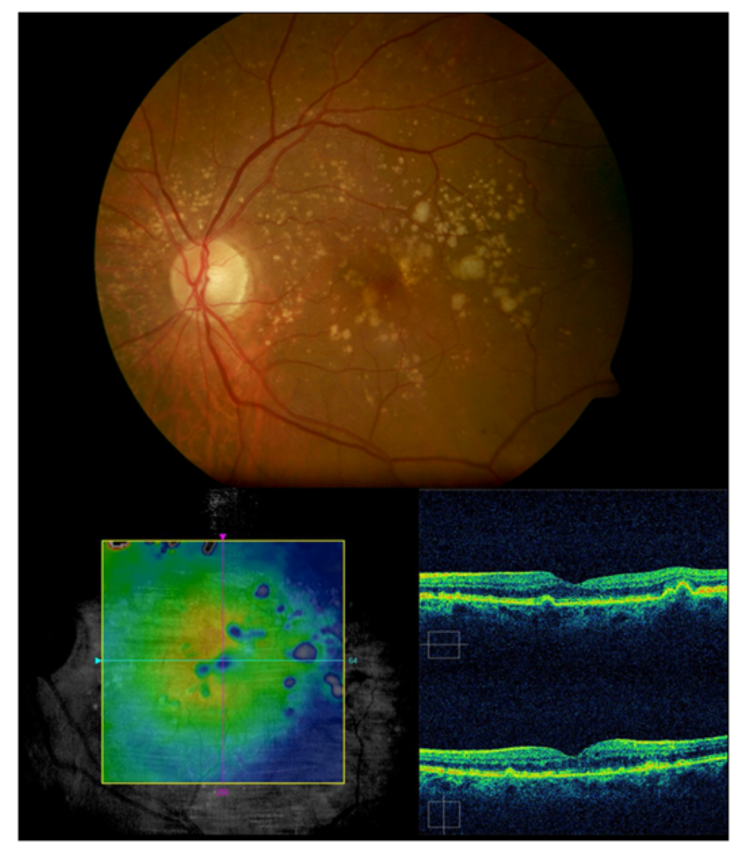

Figure 6 Cirrus $^{\mathrm{TM}}$ optical coherence tomography scan.

Notes: The same patient as in Figure 5, at the final visit: the retinal pigment epithelium had returned to a normal contour, and no fluid was evident within the retina 66 weeks after a single injection of intravitreal ranibizumab (top and bottom). The best-corrected visual acuity improved to 20/20 and remained stable through the follow-up period. No visual complaint was referred. Reprinted with permission from Gallego-Pinazo R, Marina A, Suelves-Cogollos, et al. Intravitreal ranibizumab for symptomatic drusenoid pigment epithelial detachment without choroidal neovascularization in age-related macular degeneration. Clin Ophthalmol. 201 I;5:161-165. Copyright 201 I Dove Medical Press. ${ }^{42}$
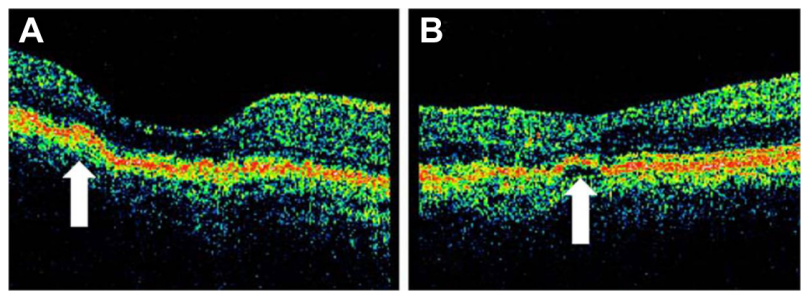

Figure 7 Stratus optical coherence tomography image.

Notes: (A and B) Comparison between drusenoid PED and soft drusen without PED. (A) Soft drusen are observed as focal elevations in the external, highly reflective band corresponding to the retinal pigment epithelium/choriocapillaris complex. (B) A drusenoid PED is a fairly well circumscribed, shallow, and often multiple elevation of the reflective band corresponding to the retinal pigment epithelium/ choriocapillaris complex.

Abbreviation: pigment PED, epithelial detachment.

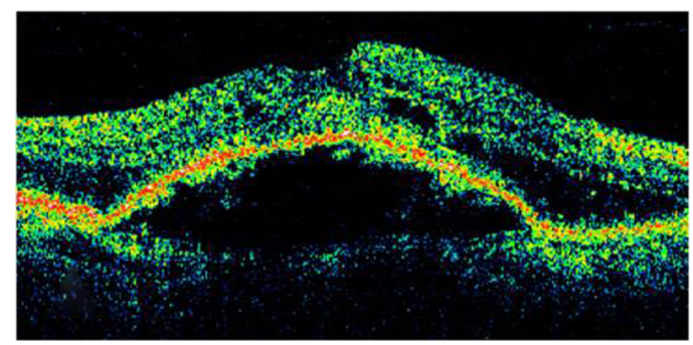

Figure 8 Stratus optical coherence tomography image.

Notes: A serous pigment epithelial detachment is observed as a smooth domed elevation of the retinal pigment epithelium, with a characteristic sharp angle of pigment epithelial detachment over an optically empty space bound inferiorly by a visible Bruch's membrane. Subretinal and intraretinal fluid is observed.

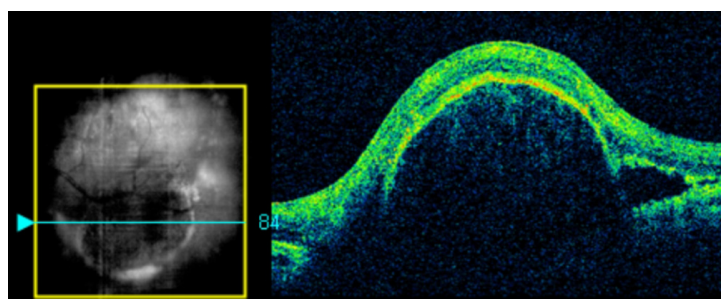

Figure 9 In this Cirrus ${ }^{\mathrm{TM}}$ optical coherence tomography image, we can see the sharply demarcated classic edges of the dome on a pigment epithelial detachment.

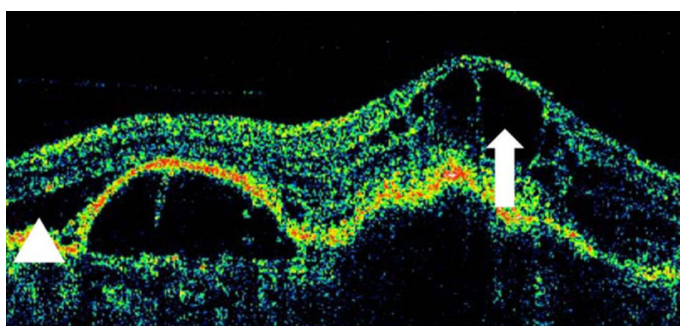

Figure 10 Stratus optical coherence tomography image.

Notes: An area of active neovascularization associated with a pigment epithelial detachment may lead to an associated smaller cuff of subretinal (arrowhead) and intraretinal (thin arrow) fluid.

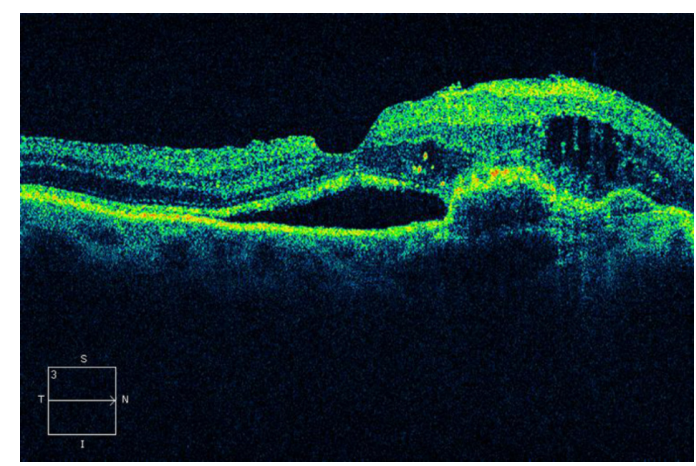

Figure II This Cirrus ${ }^{\mathrm{TM}}$ optical coherence tomography section demonstrates an elevation of the macular region with loss of the foveal depression.

Notes: The serous retinal detachment is an optically empty, black, spindleshaped zone between the retinal pigment epithelium and the neurosensory retina. Anteriorly, the retina is thickened and infiltrated with several central cysts. Posteriorly, a pigment epithelium detachment can be seen, suggesting the presence of an occult choroidal neovascularization.

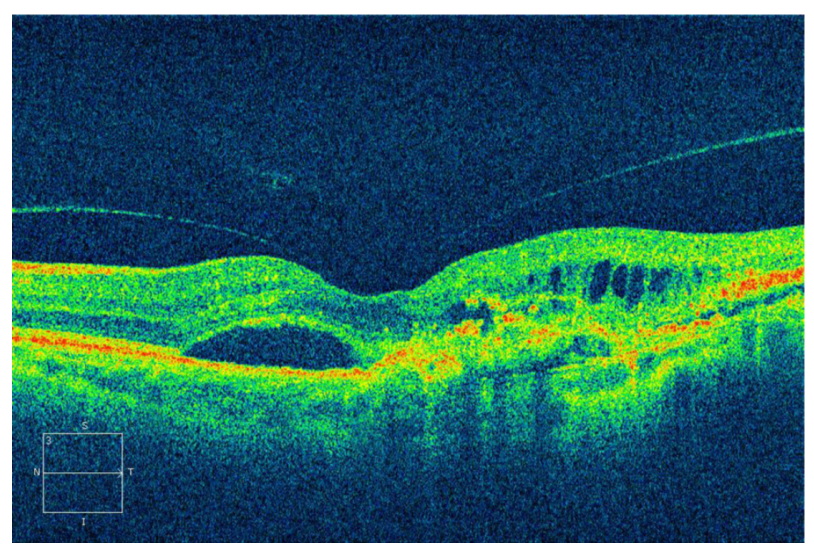

Figure 12 The combination of a pigment epithelium detachment with accompanying subretinal fluid and intraretinal fluid may be a sign of choroidal neovascularization or retinal angiomatous proliferation, in this Cirrus ${ }^{\mathrm{TM}}$ optical coherence tomography image. 
are almost always accompanied by overlying intraretinal cystic fluid. ${ }^{35}$

Certain OCT characteristics of the SPED may also help determine whether the PED is related to AMD or central serous chorioretinopathy (CSCR), another condition associated with serous RPE elevation and uncommonly associated with CNV. In a retrospective comparison of 100 eyes with either CSCR or neovascular AMD, Cho et al found that AMD eyes could be distinguished by the presence of intraretinal fluid $(P<0.0001)$, greater PED height $(354 \pm 35 \mu \mathrm{m}$ in AMD vs $187 \pm 39 \mu \mathrm{m}$ in CSCR $)(P<0.002)$, and alterations of the highly reflective line (irregularity, thickening, and attenuation) seen on TD-OCT $(P<0.0001){ }^{44}$

Hemorrhagic PEDs (HPEDs) occur when a CNV membrane bleeds into the sub-RPE space or as a result of an RPE tear. The hemorrhage may also invade the subretinal space, with sub-RPE blood having a typically darker appearance than subretinal blood. A hemorrhage associated with PED increases the suspicion for an occult CNV not seen on exam or OCT. The blood may also be located in both the subretinal and sub-RPE layers. The challenge in the treatment of HPEDs is that the CNV lesion cannot be directly visualized due to the presence of overlying hemorrhage. HPEDs have a similar ophthalmoscopic character to SPEDs, with a smooth, domed, and well-demarcated elevation but with a dark gray or black color, indicating the presence of blood. ${ }^{36}$ In contrast to the other PEDs, the imaging of HPEDs with FA will show blockage through all phases. If an associated CNV is located completely beneath the blood, it may not be visualized by FA, due to blocking.

As with SPEDs, HPEDs will often show a dome-shaped elevation of the RPE external reflective band; however, because of the optical opacification secondary to the highly pigmented and, therefore, reflective hemorrhage, the deepest posterior structures, such as the choroid, are blocked and not visualized. Therefore, subretinal hemorrhage leads to significant shadowing of the posterior layers. Occult CNV is more commonly identified on OCT as a fibrovascular PED. The fibrovascular PED is not the typical dome-shaped elevation of the RPE/BM/choriocapillaris external band seen with SPED. The elevations are often irregular, and the interior of the detachment is often filled with backscattering material, consistent with the fibrous nature of the PED. Subretinal fluid appears as an optically transparent area geographically close to the $\mathrm{CNV}$, between the RPE/BM/choriocapillaris band and the posterior edge of the neurosensory retina.

\section{Neovascular age-related macular degeneration}

Although no definitive consensus exists for the histopathologic and clinical correlation of classic and occult CNV to the
Gass classification of type I and type II membranes, some evidence exists that classic CNV is more commonly type I and that occult CNV is more commonly type II (Figure 13). ${ }^{45}$

The advances in AMD treatment with anti-VEGF therapy have largely diminished the importance of the traditional fluorescein categories of $\mathrm{CNV}$. OCT can be used to differentiate classic from occult CNV. Classic choroidal neovascular membranes typically show a diffusely backscattering fusiform thickening in the RPE/BM/choriocapillaris external band, in the geographic area where the membrane is seen. It is characterized by an increased optical reflectivity of the RPE or disruption of the highly reflective band layer RPE/choriocapillaris (Figure 14). Sometimes, the parts of the external band may appear redundant or duplicated. Subretinal fluid and intraretinal fluid, in the form of cystoid macular edema (CME), is often present (Figure 15). ${ }^{46}$ In contrast, occult subretinal neovascularization

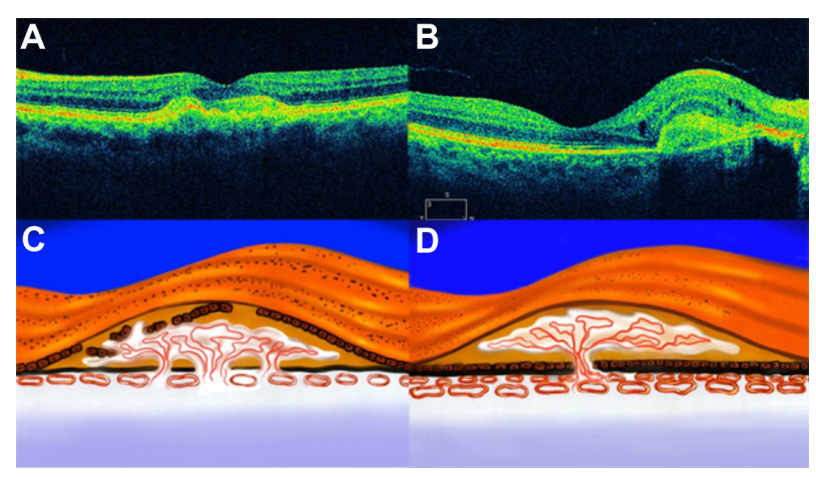

Figure 13 (A-D) The histopathologic (artist representation) and clinical correlation of occult and classic choroidal neovascularization to the Gass classification of type I (A and $\mathbf{B}$ ) and type II membranes ( $\mathbf{C}$ and $\mathbf{D})$.

Notes: $(\mathbf{A})$ and $(\mathbf{C})$ are Cirrus ${ }^{\mathrm{TM}}$ optical coherence tomography images.

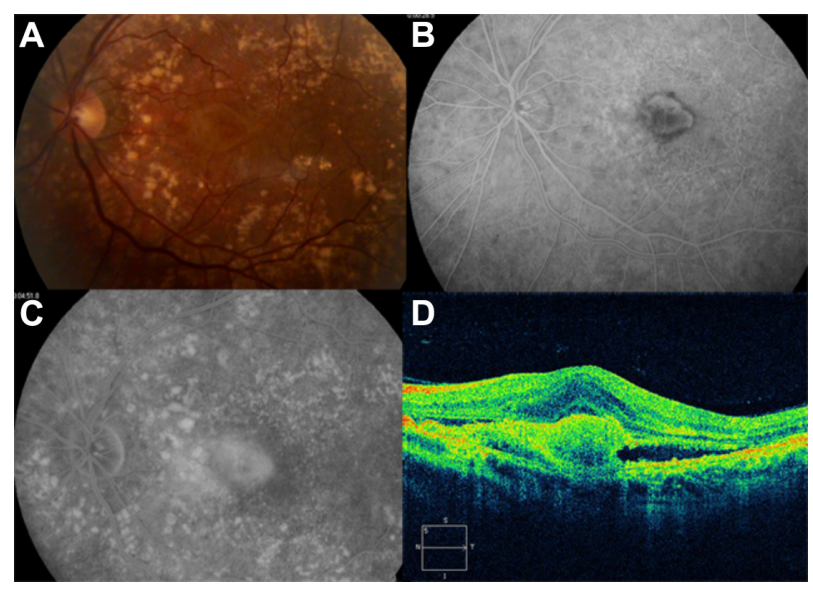

Figure I 4 Choroidal neovascularization.

Notes: (A) Fundus photography. (B) Fluorescein angiography at an early frame. (C) Fluorescein angiography at a late phase. (D) Cirrus ${ }^{\mathrm{TM}}$ optical coherence tomography image. Classic choroidal neovascular membranes typically show a diffuse backscatter and fusiform thickening in the retinal pigment epithelium/Bruch's membrane/choriocapillaris external band in the area where the membrane is seen. Choroidal neovascularization induces a bulging cone-shaped zone of hyperreflectivity accompanied by posterior shadowing. 


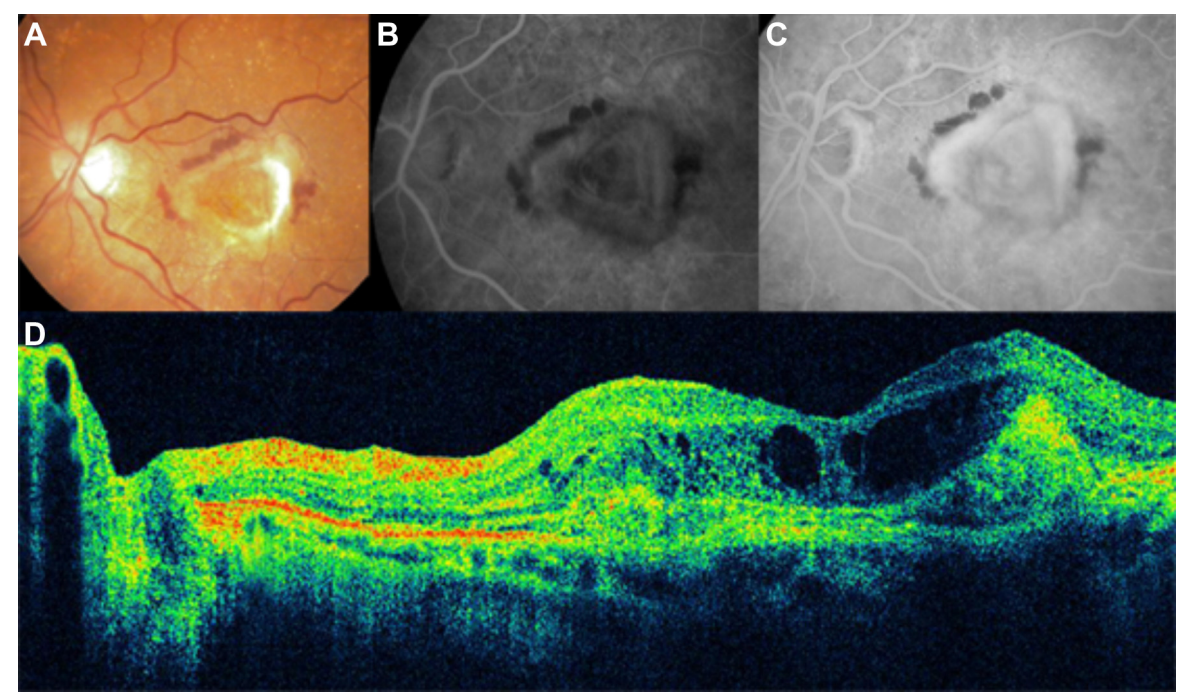

Figure 15 Choroidal neovascularization in age-related macular degeneration.

Notes: (A) Fundus photography. (B) Fluorescein angiography at an early frame. (C) Fluorescein angiography at a late frame. (D) On Cirrus ${ }^{\mathrm{TM}}$ optical coherence tomography, the central retina is thickened by many well-delineated cysts, confluent in the center and associated with diffuse intraretinal fluid accumulation.

tends to show an irregular elevation of the RPE, with a deeper area of mild backscattering, corresponding to fibrous proliferation (Figures 16 and 17). "End stage" means that the leaking or bleeding has stopped and has left a fibrous scar in the macula (Figures 18 and 19).

OCT is useful to quantitatively evaluate the differences in macular morphology in the eyes with polypoidal choroidal vasculopathy $(\mathrm{PCV})$ from the eyes with exudative AMD. ${ }^{47}$ Ozawa et al reported that serous retinal detachments (SRDs) were observed in 53\% (63/118) of the eyes with exudative AMD and in $78 \%(69 / 89)$ of the eyes with PCV $(P<0.001)$. The height of the SRD was $21.9 \pm 3.7$ microns in the eyes with exudative AMD and was $56.3 \pm 7.4$ microns in the eyes with PCV $(P<0.001)$. The thickness of the neurosensory retina was $300.0 \pm 5.2$ microns in the eyes with exudative AMD and was $275.8 \pm 4.7$ microns in the eyes with PCV $(P<0.001)$. They concluded that eyes with PCV are characterized by

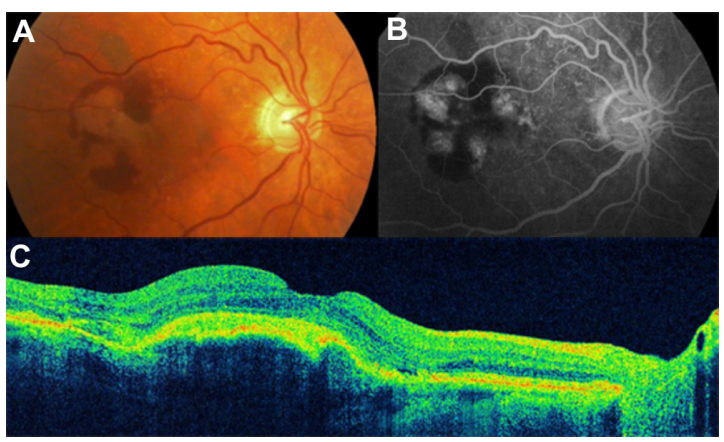

Figure 16 Choroidal neovascularization in age-related macular degeneration. Notes: (A) Fundus photography. (B) Fluorescein angiography at a late phase. (C) On Cirrus ${ }^{\mathrm{TM}}$ optical coherence tomography, occult subretinal neovascularization tends to show as an irregular elevation of the retinal pigment epithelium, with a deeper area of mild backscatter corresponding to fibrous proliferation.

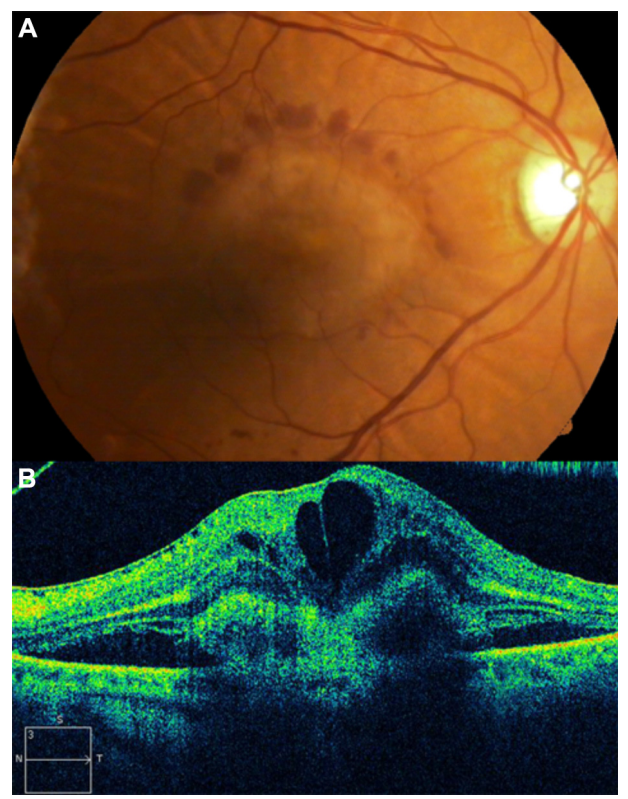

Figure 17 Choroidal neovascularization in age-related macular degeneration. Notes: (A) Fundus photography of subretinal neovascularization. (B) Cirrus ${ }^{\mathrm{TM}}$ optical coherence tomography reveals a large subretinal choroidal neovascularization with subretinal and intraretinal fluid in the macular area.

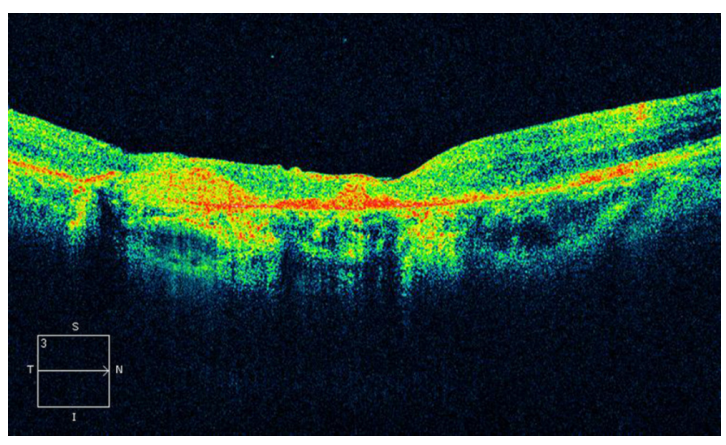

Figure 18 Cirrus $^{\mathrm{TM}}$ optical coherence tomography image shows a hyperreflective band, suggesting residual fibrosis of classic choroidal neovascularization. 


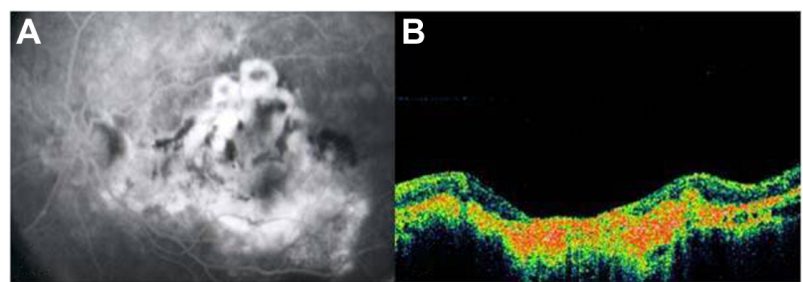

Figure 19 Disciform scar in age-related macular degeneration

Notes: (A) Angiography showed a scarred appearance of the lesion. (B) Stratus optical coherence tomography image showed an extensive fibrotic tissue with a complete atrophy of the outer and inner retinal layers in the macular area.

a higher incidence of SRDs, greater SRD height, and less intraretinal edema than eyes with exudative AMD. ${ }^{47}$

\section{Role of abnormal vitreomacular adhesion} in age-related macular degeneration

With OCT, the role of persistent vitreomacular adhesion in the development of numerous macular pathologies including idiopathic macular hole, vitreomacular traction syndrome, CME and diabetic macular edema, neovascularization in diabetic retinopathy and retinal vein occlusion, exudative AMD, and myopic traction maculopathy - has been established.

Although AMD primarily involves the outer retinal layers, several authors have suggested that the vitreous may possibly play a role in the pathogenesis and/or progression of AMD. A high incidence of posterior vitreous attachment was intraoperatively observed by different authors. ${ }^{48,49}$ Subsequent studies of the vitreous in AMD have been performed using ultrasound and have suggested that complete posterior vitreous detachment occurs less frequently in AMD than in the age-matched elder normal population and that a higher incidence of vitreomacular adhesion is detected in both exudative and nonexudative AMD. ${ }^{49,50}$ Hyaloid adhesion to the macula is associated with AMD and frequently causes vitreomacular traction in eyes with CNV. These tractional forces may antagonize the effect of anti-VEGF treatment and cause pharmacological resistance in a subpopulation of patients. OCT is an excellent method of diagnosis and follow up in these cases. ${ }^{51}$ Patients with a history of poorly responsive $\mathrm{CNV}$ despite aggressive anti-VEGF therapy and that show persistent attachment of hyaloid to the macula, with evidence of vitreomacular traction on OCT, could be surgically treated (Figures 20 and 21).

Mojana et $a l^{51}$ showed that there is a higher incidence of tractional configuration of the attached posterior hyaloid, in eyes with exudative AMD. Other investigators have also suggested a possible role of persistent vitreomacular adhesion in eyes with exudative macular degeneration. ${ }^{9,52}$

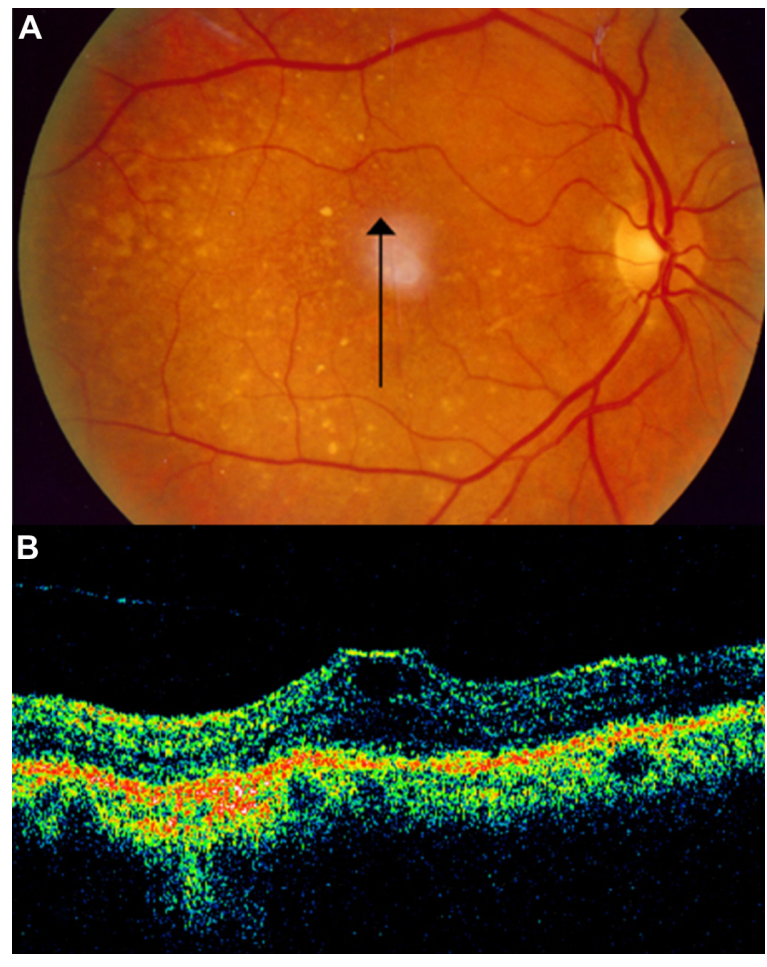

Figure 20 Vitreomacular traction syndrome and geographic atrophy. Notes: (A) Fundus photography. (B) Persistent attachment of the hyaloid to the macula, with evidence of vitreomacular traction, on Stratus optical coherence tomography.

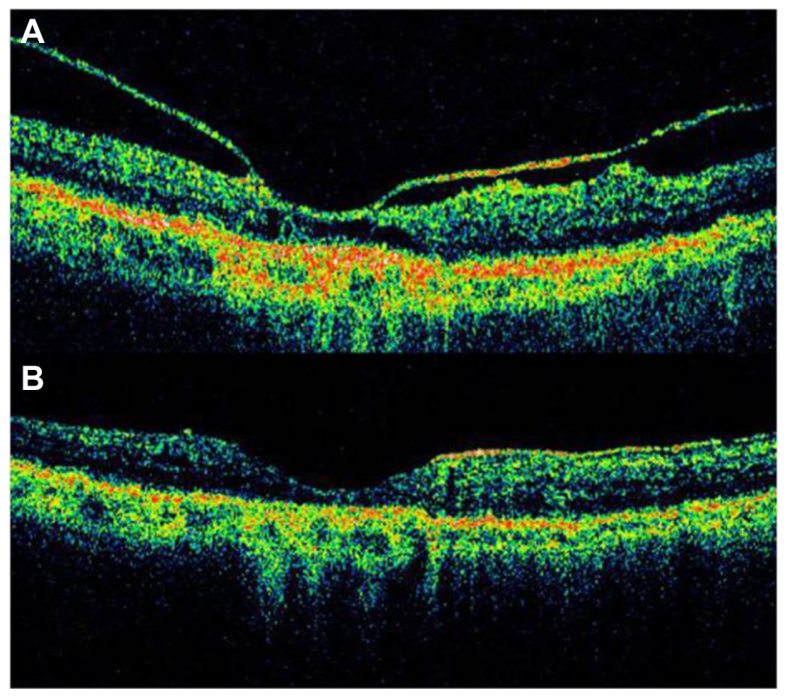

Figure 2I Vitreomacular traction syndrome and geographic atrophy. Notes: (A) Preoperative Stratus optical coherence tomography showed vitreomacular traction with attachment of the posterior hyaloid to the fovea. (B) Postoperative Stratus optical coherence tomography demonstrates relief of the vitreomacular traction.

Vitreomacular traction may cause degeneration or breaks at the RPE and BM, and as a result, the macula could be continuously exposed to the free radicals and cytokines existing in the vitreous gel, resulting in the pathogenesis and/ or progression of AMD. Posterior vitreous detachment may 
prevent continuous and direct exposure to these substances and may increase the possibility of fluid circulating behind the vitreous gel over the macular area. The rapid fluid currents not only remove the waste products and toxic materials, but also, provide oxygenation and good perfusion.

\section{Treatment of age-related macular degeneration and optical coherence tomography}

Laser treatment of subretinal neovascularization leads to atrophic scarring, which appears on OCT as highly reflective areas at the chorioretinal interface. Argon laser treatment retinal lesions evaluated in vivo by OCT has demonstrated relative high reflection in the middle of the lesion and relative low reflection around the lesion (Figure 22). ${ }^{53}$

In large multicenter studies investigating the current treatment of exudative AMD with anti-vasoproliferative substances, a fixed treatment regimen with monthly injections was previously applied. ${ }^{54,55}$ A flexible OCT-based retreatment regimen is used in new studies. The indication for retreatment is a decrease of visual acuity or an increase of retinal thickness, by OCT. Although new SD technology is available, the Stratus OCT is still the most frequently used OCT machine in these studies. Therefore, newly planned studies will need to solve the problem of different machines with different software and different values for retinal thickness that comes with the emerging SD-OCT technology.

Krebs et $\mathrm{al}^{56}$ evaluated the repeatability and reproducibility of retinal thickness measurements in exudative AMD, using the Stratus OCT. A total of 200 eyes of 200 subjects with exudative AMD were examined. The macular thickness and fast macular thickness programs of the Stratus OCT were performed twice by the same examiners or by two different examiners. The repeatability and reproducibility of the retinal thickness measurements were high, presenting better results for the macular thickness program versus the fast macular thickness program. The reliability of the retinal thickness measurement was most frequently affected by algorithm line failures and fixation problems.
SD-OCT has higher axial resolution and faster acquisition speeds compared with TD-OCT, which allow SD-OCT to provide not only the detailed views of intraretinal microstructure, but also, three-dimensional (3D) images of the macula. Sayanagi et $\mathrm{l}^{57}$ investigated the ability to delineate and detect the CNV activity in patients with exudative AMD treated with intravitreal injections of ranibizumab (Lucentis ${ }^{\circledR}$; Genentech, San Francisco, CA, USA) and found that SDOCT demonstrated a significantly better ability to delineate and detect $\mathrm{CNV}$ activity compared with TD-OCT. ${ }^{57}$

Querques et $\mathrm{al}^{58}$ studied the ability to discern the qualitative features that indicate disease activity in patients with neovascular AMD and analyzed the differences in automated retinal thickness measurement, using the Stratus TD-OCT and two different SD-OCT machines (Cirrus ${ }^{\mathrm{TM}}$ SD-OCT [Carl Zeiss] and Spectralis ${ }^{\circledR}$ SD-OCT [Heidelberg Engineering $\mathrm{GmbH}$, Heidelberg, Germany]).

SD-OCT showed a greater ability to evaluate the qualitative features indicating disease activity and had fewer errors in automated segmentation. However, the detection of central macular thickness changes was similar between TD-OCT and SD-OCT systems during follow up.

Currently, 3D scans allow the visualization of the entire scanned area, resulting in a superior ability to detect CNV activity over that of linear scans and over that of the TD-OCT radial line/fast macular thickness map scans. ${ }^{57}$ In another study, all the SD-OCT devices had higher detection rates of CNV activity after ranibizumab injections for exudative AMD than did TD-OCT. ${ }^{59}$

\section{Diabetic retinopathy Diabetic retinopathy and optical coherence tomography}

Diabetic retinopathy is a major cause of visual impairment in the United States and around the world..$^{60,61}$ Macular edema is a common cause of visual loss that occurs in up to one-third of patients with diabetes mellitus. Macular edema affects approximately $29 \%$ of diabetic patients with disease duration

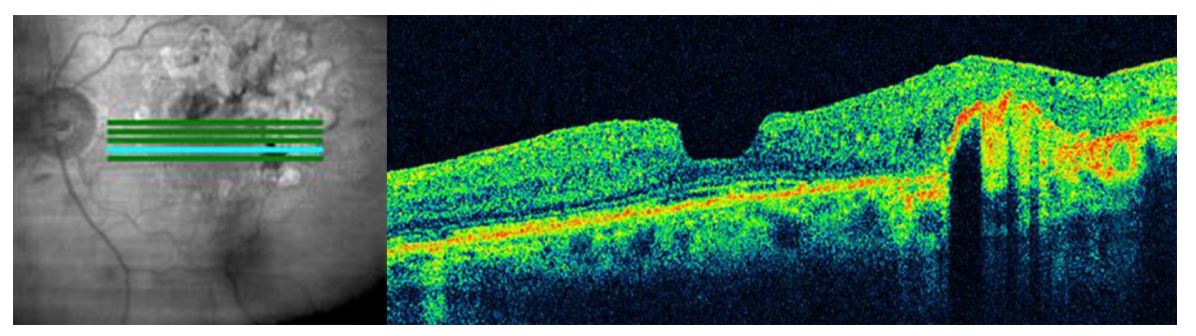

Figure 22 Laser treatment of subretinal neovascularization leads to atrophic scarring, which appears, on Cirrus ${ }^{\mathrm{TM}}$ optical coherence tomography, as highly reflective at the chorioretinal interface. 
of 20 years or more and is responsible for a significant degree of visual loss in this population. ${ }^{62}$ It involves a wide spectrum of pathologic changes, including diffuse or focal edema and $\mathrm{CME}$, which may have hard exudates. The traditional methods of evaluating macular thickening, including slit lamp biomicroscopy, indirect fundoscopy, and stereo fundus photography, are qualitative and are relatively insensitive to small changes in retinal thickness. In patients with diabetes and diabetic retinopathy, single measurements of central foveal thickness using OCT were shown to correlate with visual acuity and to be a successful means of monitoring macular thickening before and after laser therapy ${ }^{1,2}$ and/or intravitreous therapies. ${ }^{3-5}$ However, other studies report conflicting results; some studies have shown no correlation while others have shown a moderate correlation, and this issue is still in discussion..$^{5-7}$

\section{Diabetic macular edema}

OCT facilitates quantification of the retinal thickness in patients with different degrees of diabetic retinopathy, with or without clinically significant macular edema. It allows measurement of the retinal thickness from the tomograms, by means of computer image processing techniques. Objective assessment of the retinal thickness, with mapping of the posterior pole, has also been demonstrated by Gieser et al ${ }^{63}$ Baumann et al, ${ }^{64}$ and Koozekanani et al. ${ }^{65}$

OCT shows three patterns of diabetic macular edema: retinal swelling, CME, and serous retinal detachment. ${ }^{1}$ Retinal swelling shows increased retinal thickness with reduced intraretinal reflectivity and expanded areas of lower reflectivity, with cross-sectional images showing a sponge-like swelling of the retina where the low-reflective areas are expanded and where the layered structure have become irregular. Low-reflective areas are mainly located in the outer retinal layers, while the inner retinal layers are displaced anteriorly by the swollen outer retinal layers. The layered structure of the inner retinal layers is relatively preserved; however, it is occasionally interspersed with lowreflective areas (Figures 23 and 24). CME is characterized by the intraretinal cystoid spaces at the macular area. CME usually has four or five cystoid spaces located in the macula area, within a diameter of $5 \mathrm{~mm}$. In newly (within 3 months) developed CME, the cystoid spaces occupy most of the fullthickness retina, leaving thin inner and outer retinal tissue at the fovea. Perifoveal cystoid spaces are located mainly in the outer retinal layers, and the inner retinal layers are relatively preserved. Each cystoid space is walled with septa. The cystoid spaces expand in a round or oval configuration, resulting in a protruding fovea (Figure 25). In eyes with

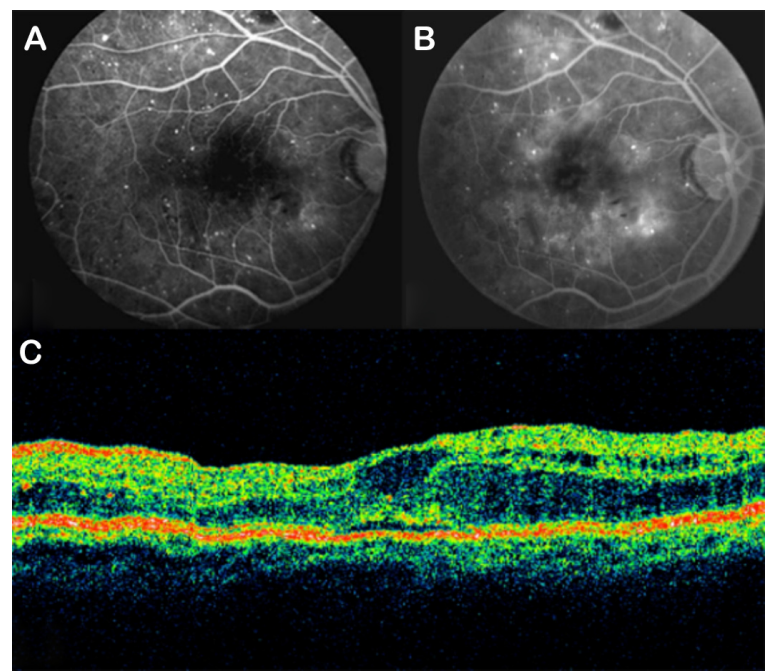

Figure 23 (A and B) Fluorescein angiography shows diffused diabetic macular edema. (C) Stratus optical coherence tomography illustrates diffuse retinal thickening and cystic spaces, consistent with cystoid macular edema.

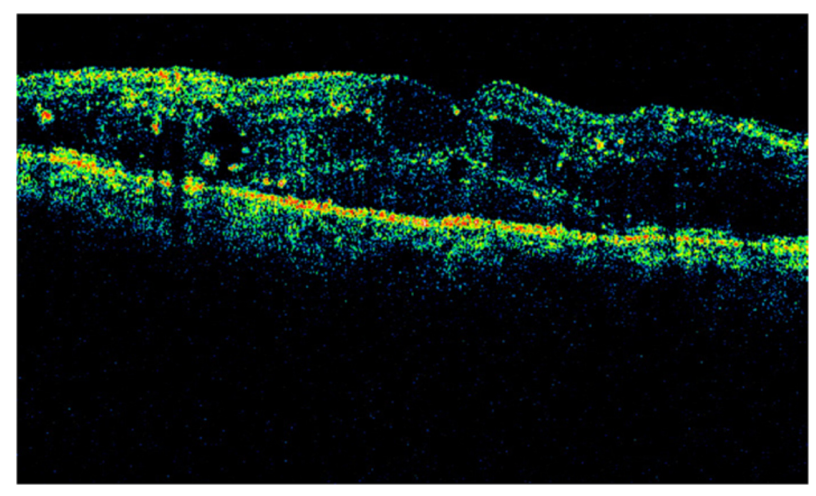

Figure 24 Stratus optical coherence tomography image. Note: The pattern of diabetic macular edema indicates retinal swelling.

well-established CME that has persisted for more than 1 year, the cystoid spaces fuse to form a large cystoid cavity (Figure 26). Serous retinal detachment is seen as a subretinal fluid accumulation with a distinct outer border of detached retina, but ophthalmoscopy sometimes fails to detect it. The thickness of the subretinal space is the greatest at the central fovea and declines peripherally (Figure 27). These basic patterns are frequently combined.

The retina is a compact tissue composed of neural elements and glial cells. Because the glial cells occupy all of the interneural space, the extracellular space is virtually absent. According to the histopathologic report in autopsied eyes with retinal edema, retinal swelling initiates in the intracytoplasmic swelling of the Müller cells. ${ }^{66}$ Sponge-like swellings in the OCT image may represent intracytoplasmic swelling of Müller cells. According to histopathologic studies, the outer plexiform layer or Henle's fiber layer is 


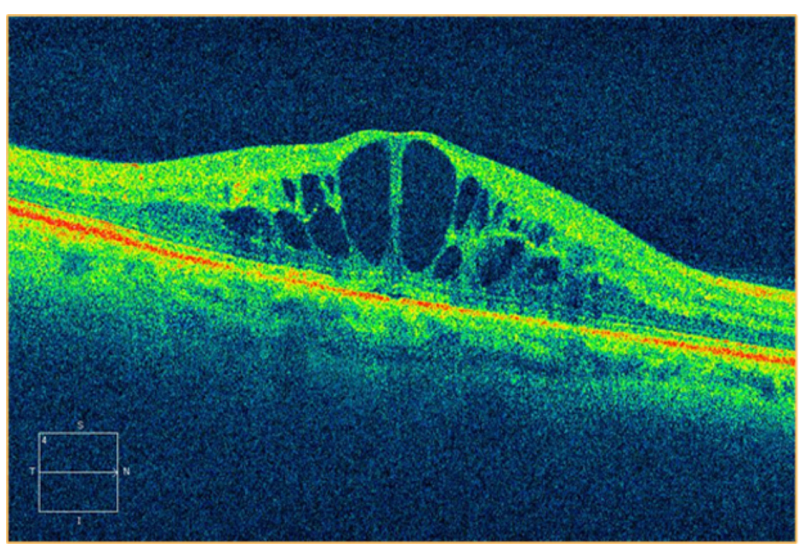

Figure 25 Cirrus $^{\mathrm{TM}}$ optical coherence tomography image.

Note: The pattern of diabetic macular edema indicates cystoid macular edema.

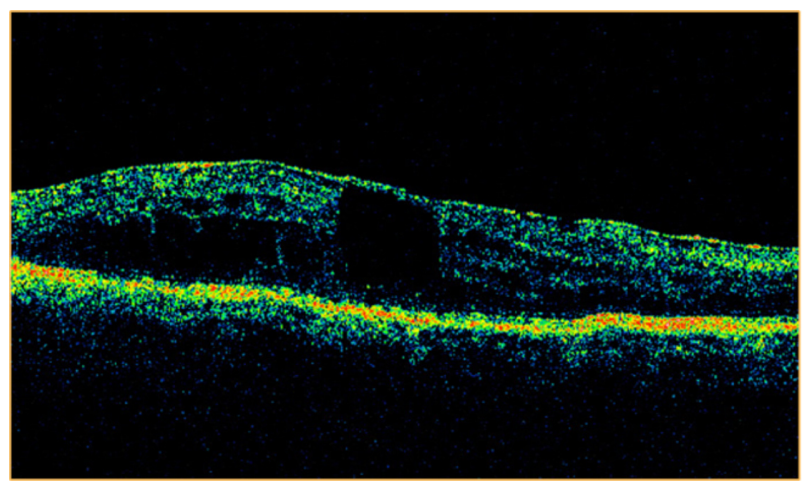

Figure 26 Stratus optical coherence tomography image.

Note: Well-established cystoid macular edema, which has persisted for more than I year, the cystoid spaces fuse to form a large cystoid cavity.

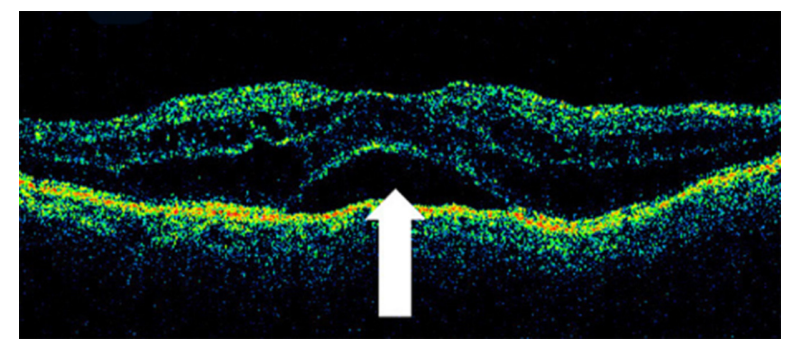

Figure 27 Stratus optical coherence tomography image.

Note: The pattern of diabetic macular edema indicates serous retinal detachment (arrow).

markedly swollen in macular edema.$^{66,67}$ A prominent space with low reflectivity in the outer retinal layers may represent the swollen Müller cells in Henle's fiber layer.

If retinal edema persists, liquefaction necrosis of the Müller cells ensues. Necrosis of the Müller cells and adjacent neural cells leads to cystoid cavity formation in the retina. ${ }^{67}$ In the histopathologic study of CME, $\mathrm{Tso}^{68}$ demonstrated that cystoid spaces were located not only in the outer plexiform layer, but also, in the inner plexiform and granular layers and even in the ganglion cell layer. Some morphologic differences were seen to exist between newly developed and well-established CME: in the former, cystoid spaces primarily were located in the outer retinal layers, and the inner retinal layers were relatively preserved; in the latter, the septa of each cystoid space disappeared, forming confluent large cystoid cavities. Large cystoid spaces sometimes involved the entire retinal layer. The remaining retinal tissue was atrophic. Chronic CME may lead to the development of a lamellar macular hole, which is evident on OCT as a partial thickness loss of retinal tissue and an abnormal retinal contour suggesting cystic rupture (Figure 28).

Hard exudates, which were located in the outer retinal layers, appeared as spots of high reflectivity with low-reflective areas behind them (Figure 29). Hard exudates increase retinal thickness in the OCT software algorithm. However, the effect of shadowing caused by exudates on the RPE is modest. Typically, a maximum increase of $5 \%$ in retinal thickness is found just beneath the hard exudate. Hemorrhage also blocks the reflections returning from the deeper retinal layers, due to the high scattering and associated high attenuation of light propagating through blood. The reflection from the AMD and choriocapillaris appeared slightly disrupted in areas of previous focal laser photocoagulation treatment (Figure 30).

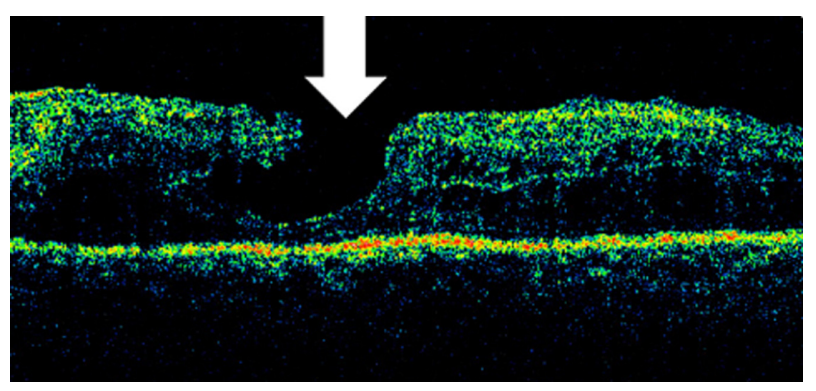

Figure 28 Stratus optical coherence tomography image.

Note: Atrophic chronic cystoid macular edema may lead to the development of a lamellar macular hole (arrow).

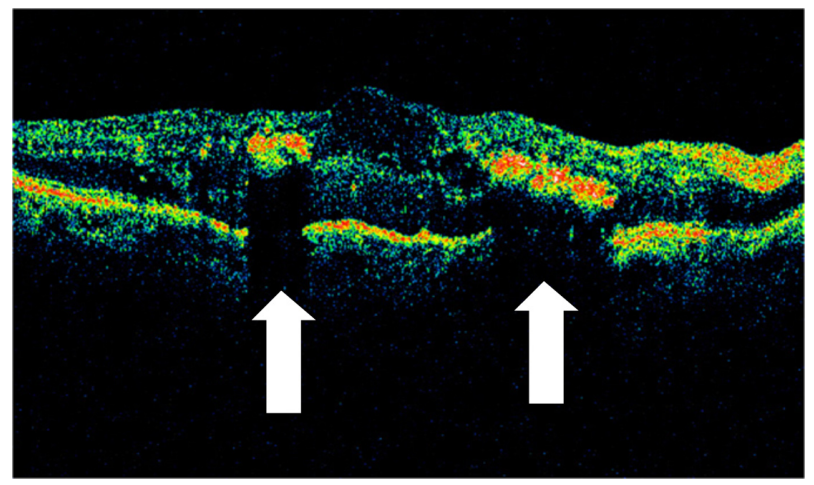

Figure 29 Stratus optical coherence tomography image.

Note: In this image, hard exudates, which were located in the outer retinal layers, appear as spots of high reflectivity with low-reflective areas behind them (arrows). 


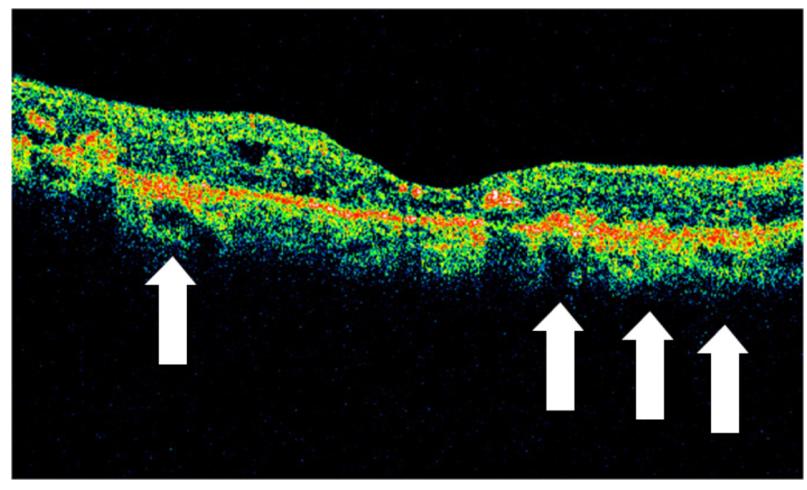

Figure 30 Stratus optical coherence tomography image.

Note: Reflection from the retinal pigment epithelium and choriocapillaris appears slightly disrupted (arrows) in the areas of previous focal laser photocoagulation treatment.

Baumann et $\mathrm{a}^{64}$ and Koozekanani et $\mathrm{a}^{65}$ showed good reproducibility of macular thickness measurement with OCT, on undilated eyes. Changes in axial length or refraction may affect measurements in the transverse direction of the eye but have no effect on measurements in the axial direction. ${ }^{65}$ Finally, a nuclear cataract does not seem to affect the retinal thickness measurement, ${ }^{69}$ although the presence of a dense subcapsular opacity may impair the ability to perform an OCT examination.

\section{Proliferative diabetic retinopathy}

Proliferative diabetic retinopathy often leads to neovascularization, hemorrhage, and preretinal fibrosis. Subhyaloid hemorrhage may occur between the inner surface of the retina and the posterior hyaloid. In proliferative diabetic retinopathy, a subhyaloid hemorrhage may result from fragile neovascularization following partial or total posterior vitreous detachment. SD-OCT shows not only the extent of the lesion relative to the fundus photograph, but also, the change in appearance of the hemorrhage in the different parts of the retina. ${ }^{70} \mathrm{~A}$ subhyaloid hemorrhage absorbs and reflects much of the OCT light and hence, creates a large shadow obscuring the underlying structures (Figure 31). Preretinal membranes are visible, in cross section, as thin, reflective bands anterior to the retina (Figure 32). Retinal traction and detachment are often present, and their extent can be directly measured from the OCT tomograms (Figures 33 and 34). The distinction between preretinal fibrosis and a detached posterior vitreous is made on the basis of reflectivity (Figure 35). The posterior hyaloid typically has a lower reflectivity than a preretinal membrane, due to the optical transparency of the vitreous. "Cotton wool" spots, due to retinal ischemia, appear in OCT cross section as regions of increased reflectivity of

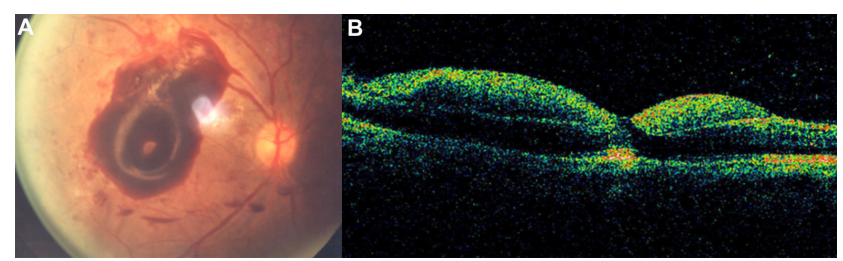

Figure 3 I (A and B) Subhyaloid hemorrhage absorbs and reflects much of the Stratus optical coherence tomography light and hence, creates a large shadow obscuring the underlying structures.

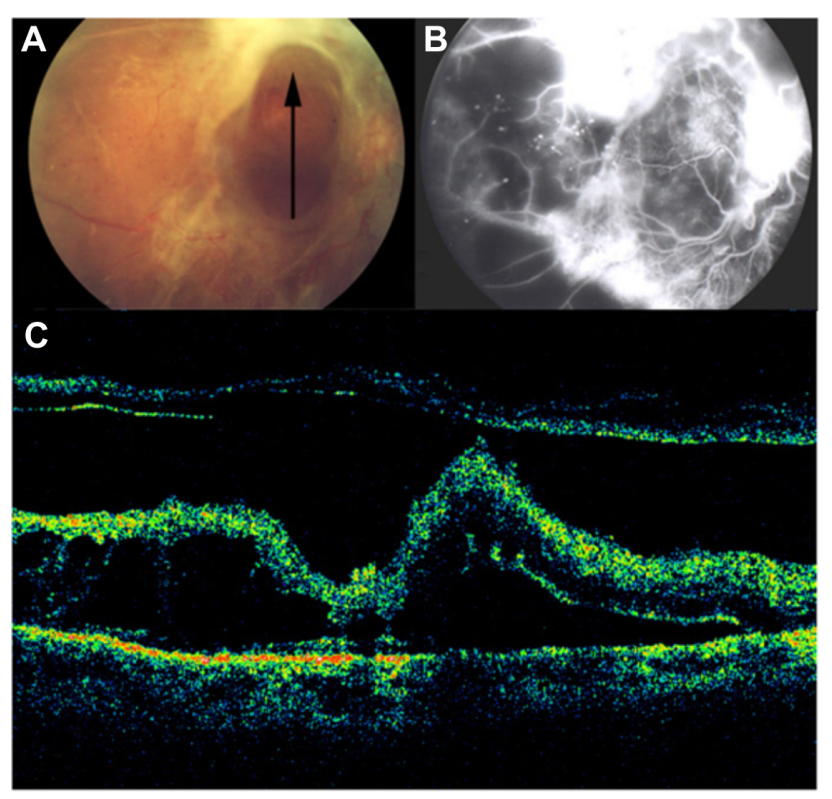

Figure 32 (A) Fundus photography, (B) fluorescein angiogram, and (C) Stratus optical coherence tomography of proliferative diabetic retinopathy with preretinal membranes (thin, reflective bands anterior to the retina) and cystoid macular edema.

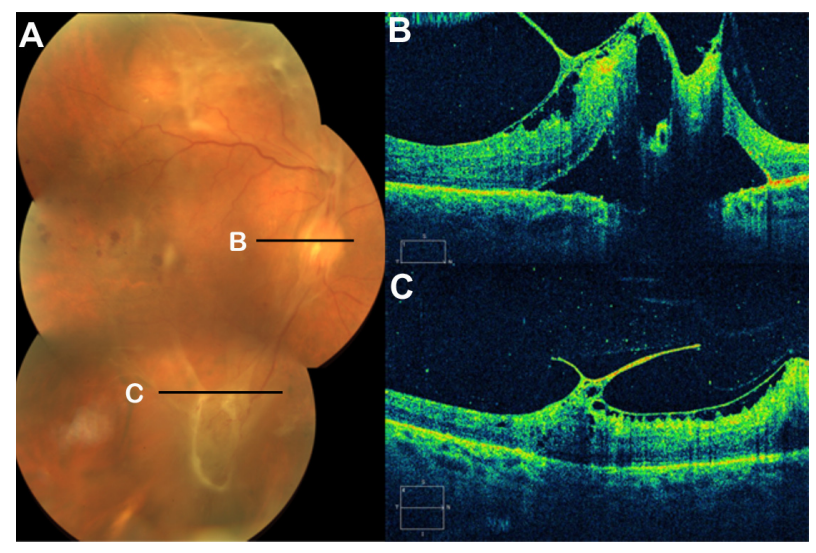

Figure 33 (A) Fundus photography, (B) fibrovascular proliferations of the optic disc (B-line), and (C) Cirrus ${ }^{\mathrm{TM}}$ optical coherence tomography of proliferative diabetic retinopathy with retinal traction and tractional detachment (C-line).

the retinal nerve fiber layer and inner neurosensory retina. Retinal thinning corresponding to retinal atrophy can be defined by OCT in the region of previous photocoagulation treatment (Figure 36). 


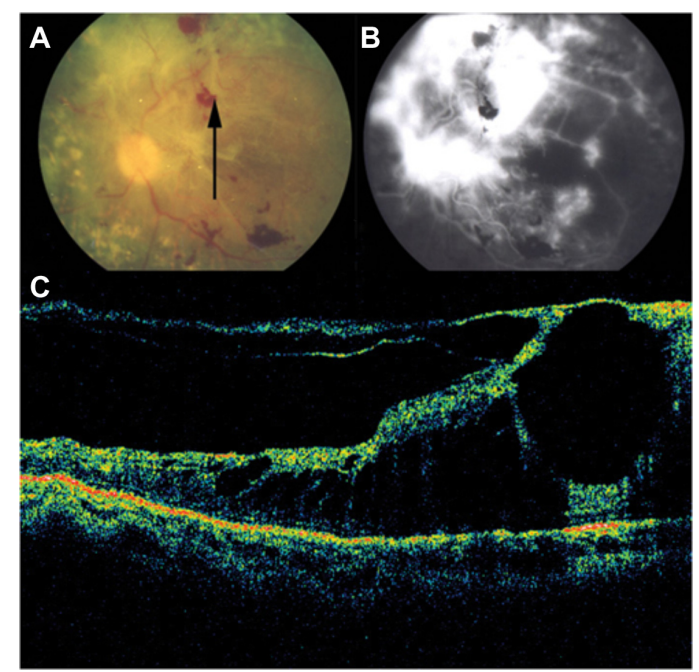

Figure 34 (A) Fundus photography, (B) fluorescein angiogram, and (C) Stratus optical coherence tomography of proliferative diabetic retinopathy with retinal traction and detachment.

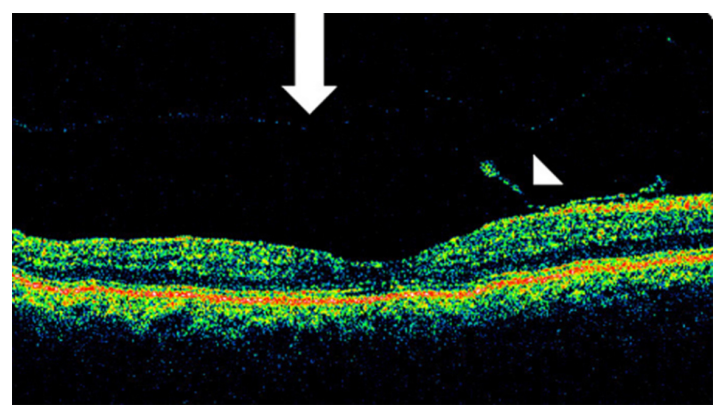

Figure 35 Stratus optical coherence tomography image.

Notes: The distinction between preretinal fibrosis and a detached posterior vitreous is made on the basis of reflectivity. The posterior hyaloid typically (arrow) has a lower reflectivity than a preretinal membrane (arrowhead).

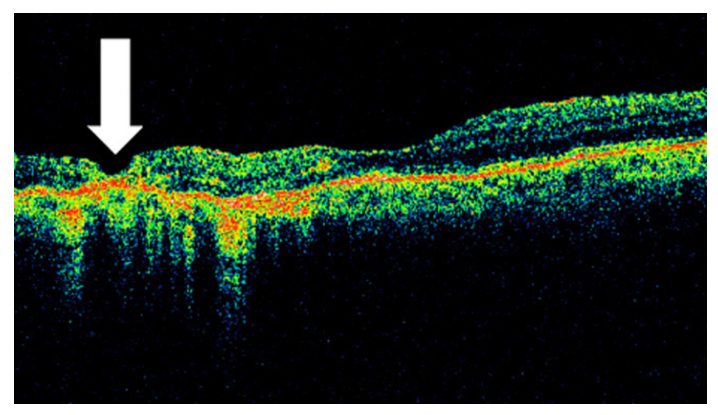

Figure 36 Retinal thinning (arrow) corresponding to retinal atrophy can be defined by Stratus optical coherence tomography, in the region of photocoagulation treatment.

\section{Diabetic tractional papillopathy}

Kroll et al ${ }^{71}$ reported partial restoration of vision following vitrectomy in 15 of 17 eyes, ostensibly through the removal of the diabetic fibrovascular proliferations from the nasal part of the optic disc (Figure 37) and the relief of vitreopapillary traction which (for between 6 months and 6 years) had caused a reversible functional impairment of the papillomacular bundle via the stretching and kinking of ganglion cell axons

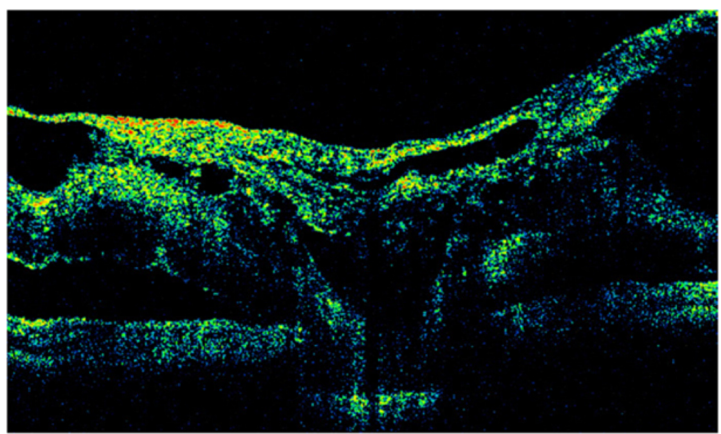

Figure 37 Stratus optical coherence tomography image.

Note: The image shows highly reflective diabetic fibrovascular proliferation of the optic disc.

and additional or consecutive effects on their prelaminar blood supply. Eyes with such features (that is, with traction primarily localized nasally on the disc and unaccountably affecting visual acuity without any associated disturbance of the central visual field) should be subjected to early vitrectomy in order to prevent irreversible long-term damage to central vision. It merits the careful attention of all ophthalmologists involved in the management of diabetic eye disease.

OCT can be useful to determinate the vitreous state; OCT can determine whether the vitreous is attached or detached from the retina and the optic disc (Figure 38). With

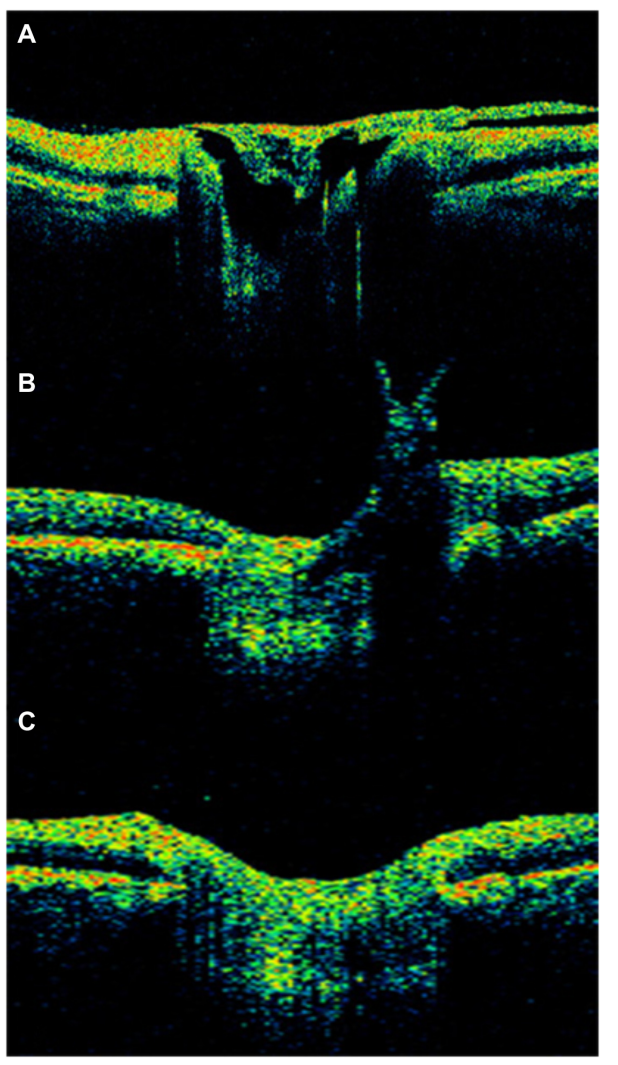

Figure 38 Stratus optical coherence tomography can determine the vitreous state. Notes: The images show (A) fibrovascular proliferations of the optic disc; (B) thickening of the posterior hyaloid, which is especially hyperreflective and is attached to the optic disc; and (C) the optic disc without vitreous traction. 
vitreopapillary traction, the thickened posterior hyaloid is especially hyperreflective and is attached to the disk. Also, the thickened posterior hyaloid exerts tangential and/or anteroposterior vitreopapillary traction.

\section{Other retinal pathologies \\ Central serous chorioretinopathy}

CSCR is characterized by serous detachments of the neurosensory retina in the macular region (Figures 39 and 40). This is a chorioretinal disease commonly seen in young and middle-aged men. Patients often develop one or more small areas of AMD serous detachment in the macula or in the paramacular area, clearly seen in the OCT image (Figures 41 and 42). ${ }^{72,73}$

Gass $^{74}$ postulated that an increase in the permeability of the choriocapillaris resulted in PED and exudation of fluid

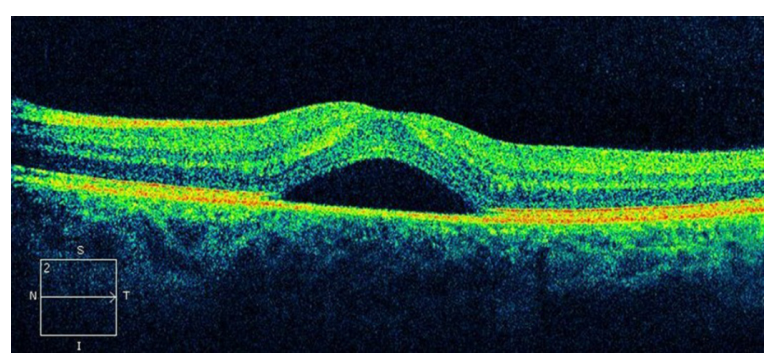

Figure 39 Cirrus $^{\mathrm{TM}}$ optical coherence tomography.

Note: Central serous chorioretinopathy is characterized by serous detachments of the neurosensory retina in the macular region.

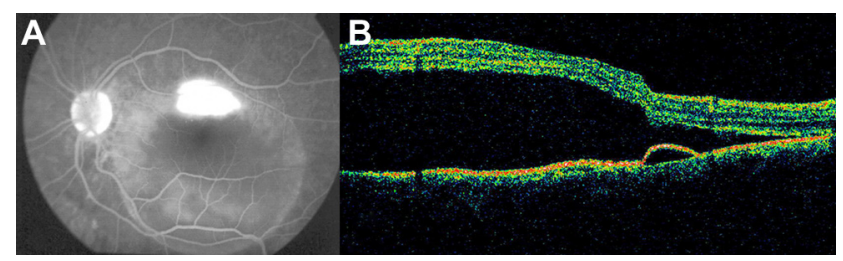

Figure 4 I Central serous chorioretinopathy.

Notes: (A) Fluorescein angiography demonstrating the leakage site at the retinal pigment epithelium, and neurosensory retinal detachment. (B) Stratus OCT demonstrates a pocket of serous fluid elevating the neurosensory retina. At the edge of this retinal detachment, there is a focal elevation of the retinal pigment epithelium over a clear space. This corresponds to the small area of retinal pigment epithelium serous detachment in the paramacular area, clearly seen in the OCT image.

Abbreviation: OCT, optical coherence tomography.

into the subretinal space. Studies of CSCR using indocyanine green angiography have shown multiple areas of choroidal vascular hyperpermeability, ${ }^{75-78}$ vascular congestion, and venous dilation, ${ }^{78-80}$ suggesting that a generalized choroidal vascular disturbance occurs in CSCR.

The gold standard for CSCR diagnosis is FA, which displays subretinal fluid accumulation, RPE detachment, and typical dye leakage from the choroid into the subretinal space. ${ }^{72}$ Although FA is more accurate in assessing the retinal lesions, the invasiveness of this technique may hinder its repeated use in the long term. Therefore, noninvasive diagnostic systems, such as OCT, may be used as alternative diagnostic tool.

Hirami et $\mathrm{al}^{81}$ compared the findings by OCT and FA in 30 eyes. They found that 22 of the 25 eyes with acute CSCR showed OCT abnormalities in the RPE (88\%). These abnormal-

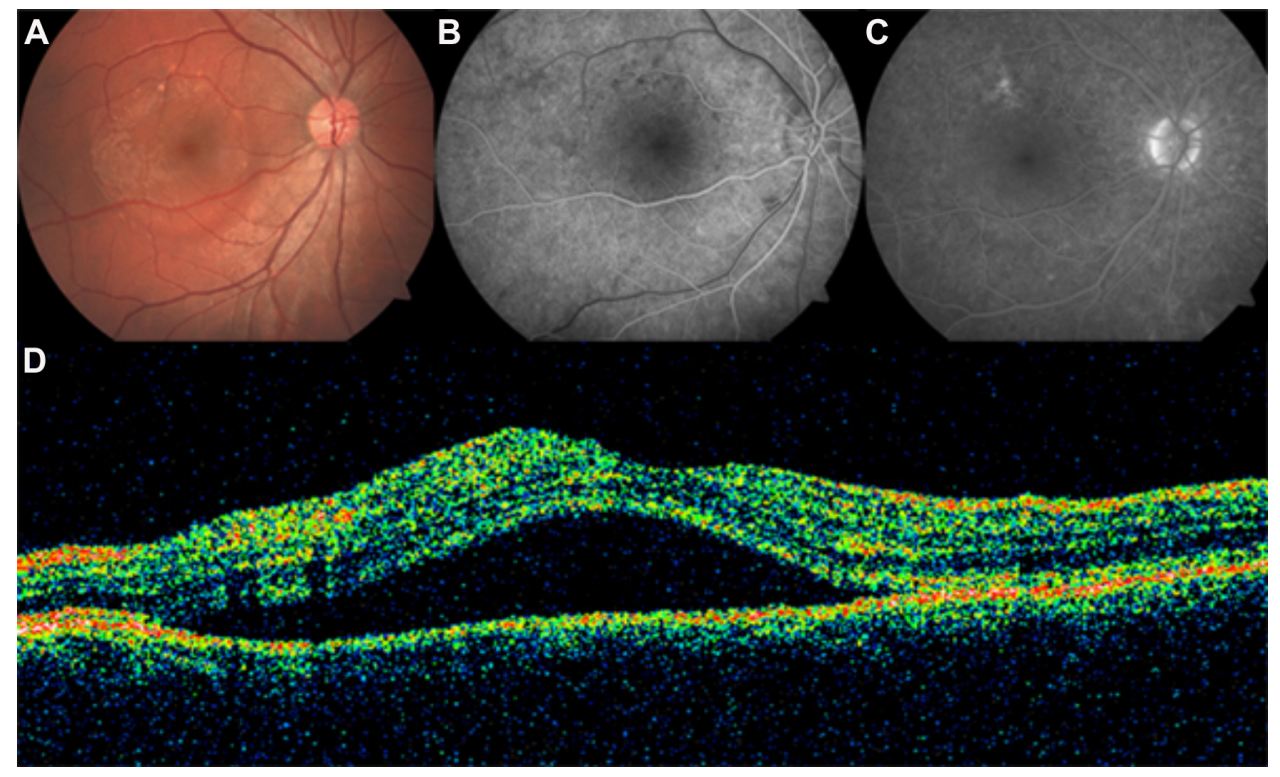

Figure 40 Central serous chorioretinopathy.

Notes: (A-C) Color photograph and fluorescein angiography early and late frames. (D) Stratus optical coherence tomography shows serous detachments of the neurosensory retina in the macular region. 


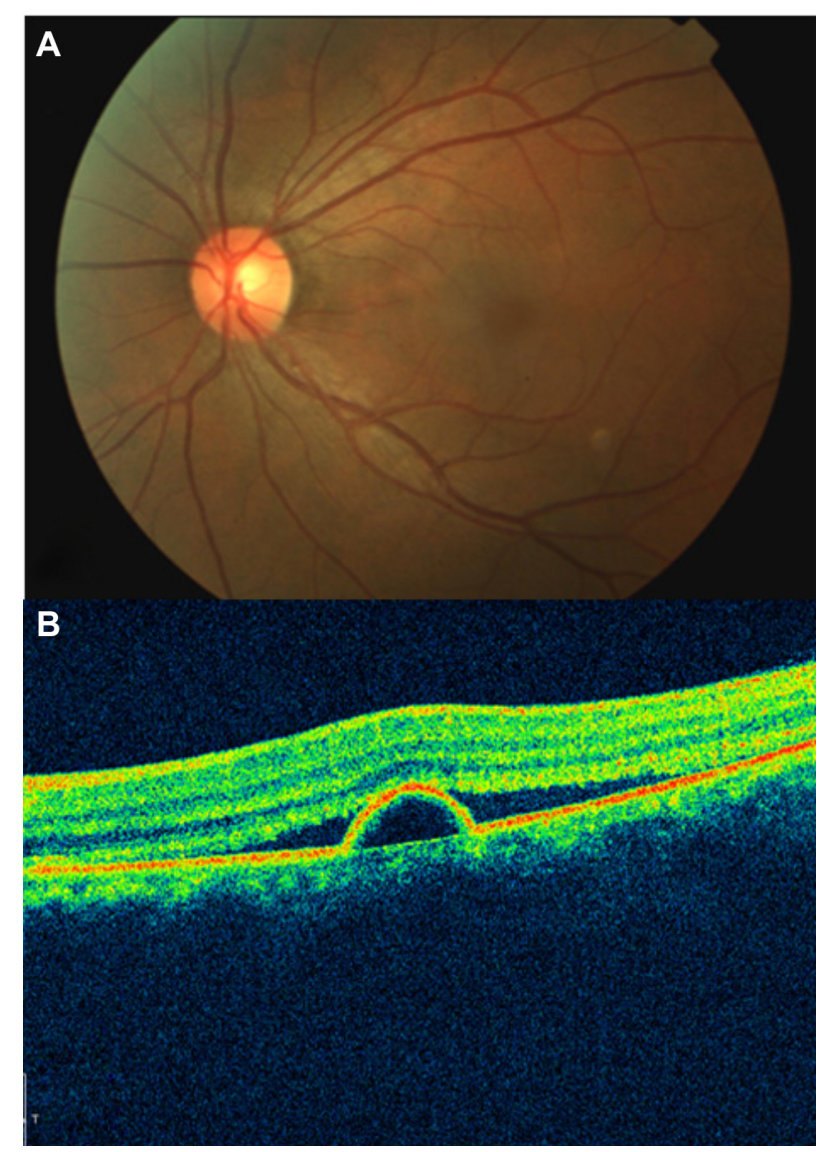

Figure 42 Central serous chorioretinopathy.

Notes: (A) Color fundus photograph in central serous chorioretinopathy. (B) Retinal pigment epithelium serous detachment and neurosensory retinal detachment are clearly observed on this Cirrus ${ }^{\mathrm{TM}}$ optical coherence tomography image.

ities included RPE detachment in nine eyes (36\%) and a small bulge on the RPE at the posterior pole in 15 eyes (60\%). Of the 29 eyes with acute or chronic CSCR, FA showed characteristic pinpoint leakage in 20 eyes $(69 \%){ }^{81}$ Another recent study on SD-OCT examination revealed that the OCT scan can reveal a leakage site through which fluid may pass to the subretinal area. ${ }^{82}$ The authors concluded that SD-OCT may offer new information to facilitate the understanding of the mechanisms of acute CSCR. ${ }^{82}$ Recently, Wang et al ${ }^{83}$ demonstrated that a combination of 3D OCT and fundus photochromy offered a high identification rate of the leakage spots. The combination of the two noninvasive techniques may be used as an alternative diagnostic or evaluation tool for acute CSCR.

In a neurosensory detachment, the OCT tomogram appears as a shallow elevation of the retina, with an optically clear space between the retina and the high reflection of RPE/ choriocapillaris, and the height of the cavity can be exactly measured. Examination with OCT is more sensitive to the identification of small elevations of the neurosensory retina than is slit lamp biomicroscopy. ${ }^{84}$ The detached RPE may be distinguished by a focal elevation of the higher-reflective layer over a clear cavity.

Enhanced depth imaging (EDI) SD-OCT is a noninvasive method used to image the choroid and may be a valuable modality in the diagnosis and treatment of CSCR. ${ }^{85,86}$ The EDI-OCT technique, described by Spaide et al, improves the depth imaging capabilities of SD-OCT because it shifts the peak of the sensitivity curve of SD-OCT to the inner sclera ${ }^{85} \mathrm{With}$ this additional imaging technique, Imamura et $\mathrm{al}^{87}$ demonstrated that patients with CSCR had an increased subfoveal choroidal thickness compared with patients with normal eyes. The choroid was thick in both eyes, even when subretinal fluid was seen in only one eye. This finding provides additional evidence that CSCR may be caused by increased hydrostatic pressure in the choroid.

A neurosensory detachment may be connected with an RPE detachment, by the discontinuity on the RPE. Longitudinal examinations with OCT can detect exact changes in the height measurements of subretinal fluid and can detect CSCR resolution. Montero and Ruiz-Moreno recently described that bulges protruded from the RPE under a neurosensory detachment, in acute and chronic CSCR, which probably meant activity of the disease. ${ }^{73}$ CSCR typically occurs in young healthy patients, ${ }^{88}$ but when neurosensory retinal detachment occurs in older patients ${ }^{77,89}$ this condition can be mistaken for neovascular AMD. It may be difficult to distinguish CSCR clinically, especially from the occult form of neovascular AMD, since occult CNV may have a similar angiographic appearance to CSCR. ${ }^{84}$ Neovascular AMD and acute CSCR have distinguishing features on OCT that may help clinicians to differentiate accurately between the two conditions, primarily using OCT. ${ }^{44}$

Cho et $\mathrm{al}^{44}$ demonstrated that the presence of intraretinal fluid, large PEDs, and alterations of the highly reflective line, characterized by irregularity, thickening, and attenuation, was significantly more common in neovascular AMD than in acute CSCR. On the other hand, acute CSCR was notable for having significantly more and larger subretinal fluid and hypertrophic outer retinal changes, but the highly reflective line remained regular in thickness and morphology. In their study, they encountered numerous patients in an intermediate age range (55-70 years) with accumulations of submacular fluid and without frank hemorrhage that were difficult to diagnose with certainty. FA is typically not diagnostic in these circumstances. The OCT characteristics, especially the lack of disruption at the highly reflective line 
(in CSCR), the presence of intraretinal fluid (in AMD), and the greater height of subretinal fluid (in CSCR), are distinguishing features that appear to be highly reliable. ${ }^{44}$

In conclusion, in CSCR, OCT can provide additional information and may help distinguish CSCR from subretinal neovascularization and other macular diseases. OCT has been shown to be superior to biomicroscopy and FA in identifying CSCR. ${ }^{90}$

\section{Retinal vascular occlusive disease}

Retinal vascular diseases are common etiologies for central visual loss. Central retinal artery occlusion is an emergent ocular problem that frequently results in profound and permanent loss of vision. Pathologically, the inner retinal cells soon become edematous after occlusion of the artery. It is well known that central retinal artery occlusion causes an initial ischemic necrosis with intracellular edema at the acute stage and later results in the formation of an acellular scar of the inner retinal cell layers. ${ }^{91}$

In patients with branch retinal artery occlusion and central retinal artery occlusion, OCT shows increased retinal thickness with cystic spaces of low reflectivity. In addition, the inner retinal layers tend to be more highly reflective in OCT, probably due to the ischemia of these layers. Therefore, this high reflection causes shadowing of the optical signal of the RPE/ choriocapillaris complex. The increased thickness of the inner retina typically seen in acute artery occlusions corresponds to the intracellular edema (Figure 43). OCT complements the information obtained from FA in retinal vascular diseases.

Occlusion of the retinal venous system is the second most common retinal vascular disease after diabetic retinopathy. ${ }^{92}$ In branch retinal vein occlusion (Figure 44) and central retinal vein occlusion (Figures 45 and 46), OCT findings include CME, serous retinal detachment, epiretinal membranes, pseudoholes, lamellar holes, and subhyaloid or preretinal hemorrhages. A significant proportion of patients with occlusion of the retinal venous system have OCT evidence of CME and serous retinal detachment. OCT shows hyporeflective intraretinal cavities, in cross-sectional scan, radiating from the center of the macula in CME. Macular edema is associated with serous retinal detachment in retinal venous system occlusion. ${ }^{92}$ OCT plays a pivotal role in quantitatively monitoring the changes in retinal thickness after treatment, such as intravitreal triamcinolone or anti-VEGF therapy (Figure 47). ${ }^{93}$

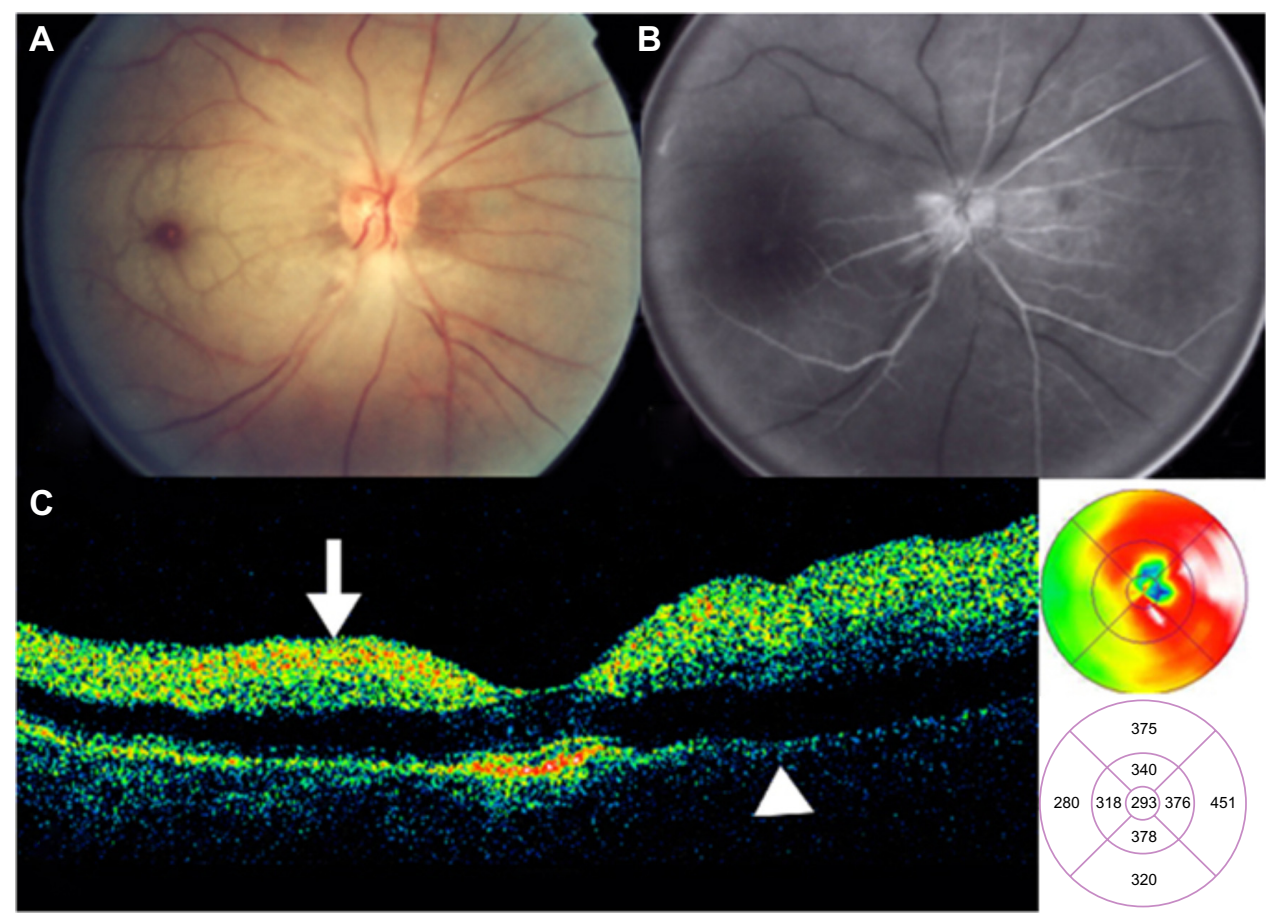

Figure 43 Central retinal artery occlusion.

Notes: (A) Color fundus photograph demonstrating whitening of the retina and a "cherry-red" spot. (B) Fluorescein angiogram reveals poor retinal vascular filling. The leading edge of dye within the superior arterial system is distinctly abnormal and indicates hypoperfusion. (C) Stratus optical coherence tomography image: the horizontal optical coherence tomography scan shows increased thickness and reflectivity of the inner retinal layers (arrow); this high reflectivity causes shadowing of the optical signals of the outer retinal layers and the retinal pigment epithelium/choriocapillaris complex (arrow head). The retinal thickness map demonstrates increased central thickness (293 microns), especially nasal to the fovea, due to intracellular edema and ischemia of the papillomacular bundle (insert). 


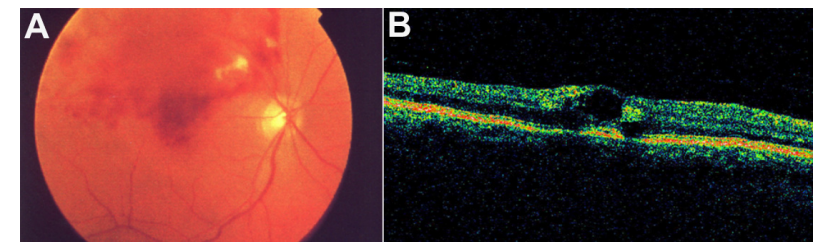

Figure 44 Branch retinal vein occlusion with macular edema.

Notes: (A) Fundus photograph. (B) Stratus optical coherence tomography image. The horizontal optical coherence tomography scan showed diffuse retinal thickening and low-reflective spaces consistent with macular cysts.

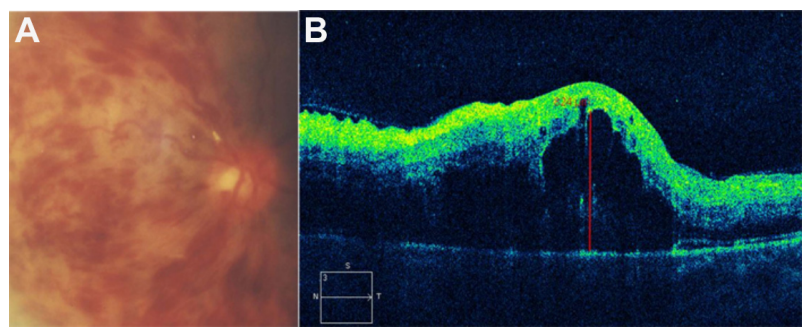

Figure 45 Central retinal vein occlusion with macular edema.

Notes: (A) Fundus photograph. (B) The Cirrus ${ }^{\mathrm{TM}}$ optical coherence tomography scan showed a thickened elevated macula with numerous low-reflective cystic spaces representing fluid accumulation. A detachment of the neurosensory retina, with subretinal fluid accumulation is observed underneath the fovea.
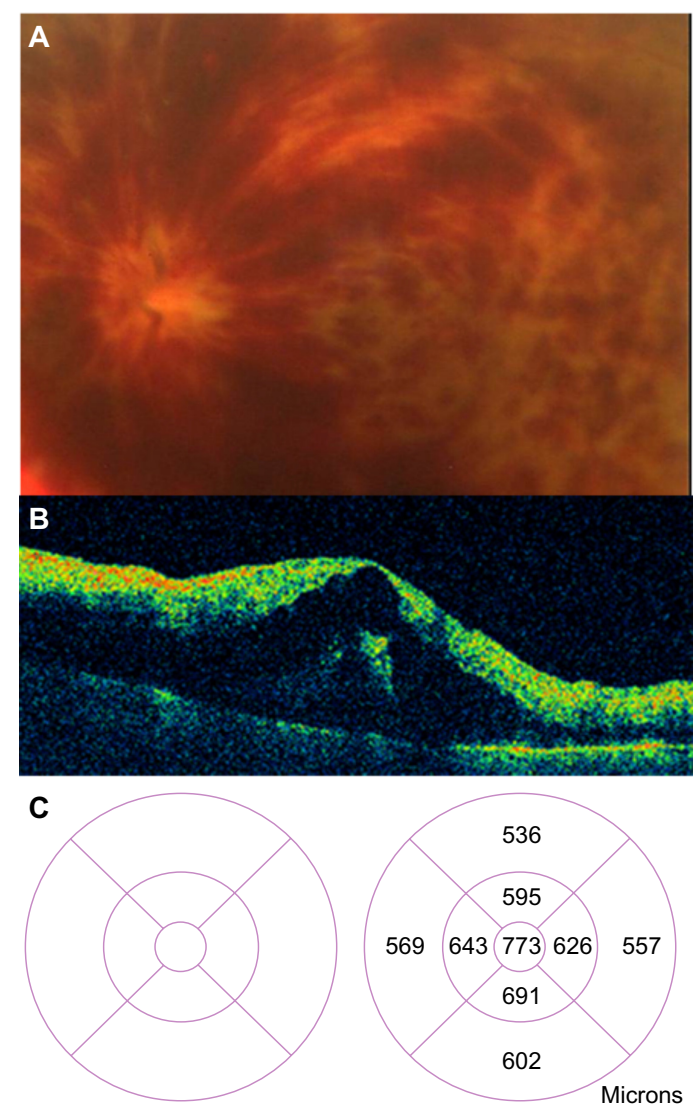

Figure 46 Acute central retinal vein occlusion with macular edema.

Notes: (A) Fundus photograph. (B) Horizontal Stratus optical coherence tomography scans showed an elevated macula with diffuse retinal thickening and low-reflective spaces consistent with macular cysts and intra-subretinal fluid. (C) The retinal thickness map demonstrates increased central thickness (773 microns).

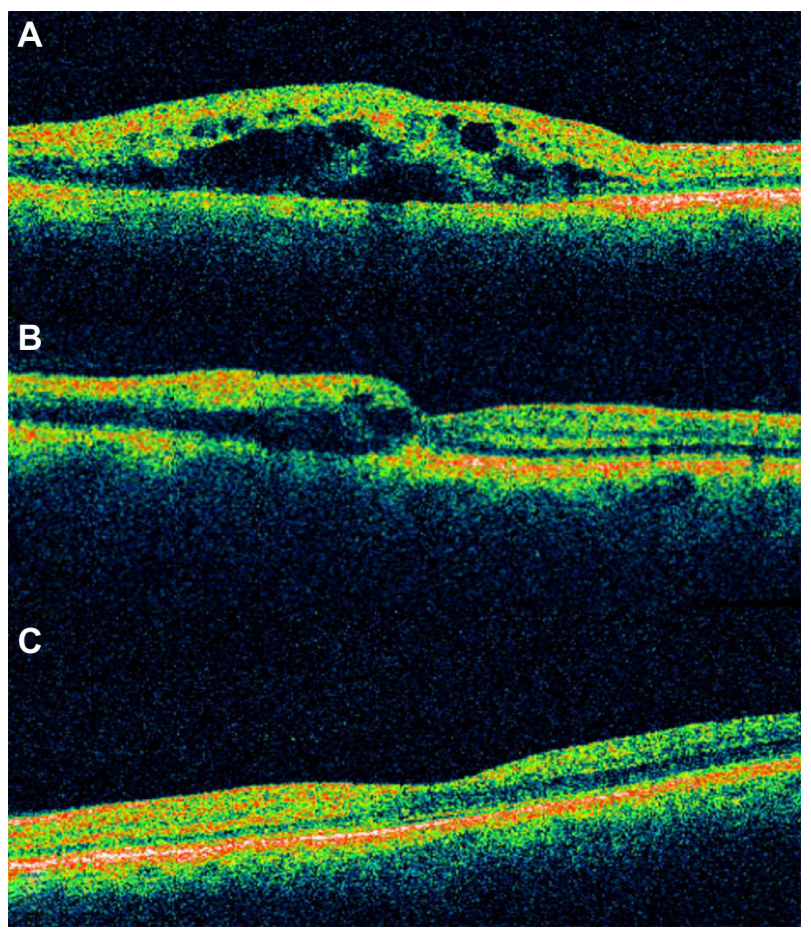

Figure 47 (A) Acute branch retinal vein occlusion with macular edema: the horizontal Stratus optical coherence tomography scan showed numerous hyporeflective spaces within the outer plexiform layer; intraretinal thickening leads to a loss of foveal contour. (B) A decrease of central macular thickness was observed I week after intravitreal bevacizumab therapy. (C) A normal macular thickness was seen 2 months after initial therapy.

\section{Myopic retinoschisis}

Posterior retinoschisis with high myopia is not uncommon. It may occur secondarily to posterior staphyloma, retinal atrophy, or traction from the vitreous or retinal arterioles ${ }^{94,95}$ and usually cannot be easily detected by fundoscopic examination. Therefore, the diagnosis of retinoschisis in high myopia is often delayed due to poor clinical visualization of the schisis and retinal atrophy.

SD-OCT images can help to clearly demonstrate the morphological changes of retinoschisis (Figure 48). With this technology, we can observe details within the retina and have a better understanding of this particularly macular disease. The schisis could be located at the outer, middle, or inner layers of the retina, but the outer schisis is the most commonly observed. ${ }^{96}$ It provides important evidence as to the advantage of treatment with vitrectomy with internal limiting membrane peeling and helps to explain the visual outcome by documenting the recovery of the inner segment-outer segment layer after surgery. An OCT examination should be considered in patients with high myopia and decreasing vision with no obvious fundoscopic findings. ${ }^{96}$ 


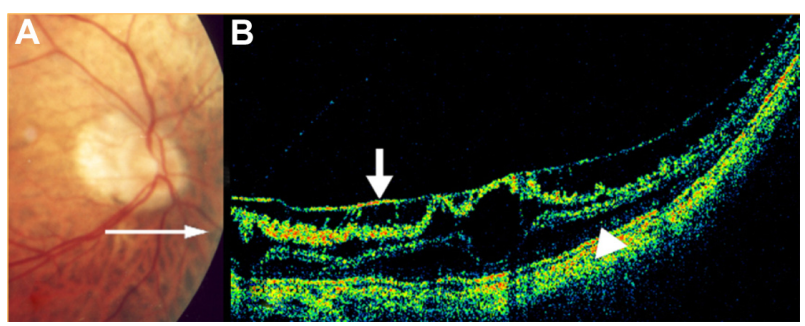

Figure 48 Myopic degeneration with retinoschisis.

Notes: (A) The color fundus photograph distinctly shows a myopic crescent as a white, sharply defined area where the inner surface of the sclera is seen. Retinoschisis inferior to the optic disc was not differentiated ophthalmoscopically. (B) A horizontal Stratus optical coherence tomography scan obtained inferior to the optic disc demonstrates a splitting of the neurosensory retina (arrow) and the separation of full-thickness neurosensory retina from the underlying retinal pigment epithelium (arrowhead).

\section{Pseudophakic cystoid macular edema}

Pseudophakic CME, also known as Irvine-Gass syndrome, is one of the most common causes of visual loss after cataract surgery. ${ }^{97,98}$ The incidence of CME varies widely, but is likely in the range of $1 \%-2 \%$, using modern cataract extraction techniques. Pseudophakic CME classically presents 4 to 6 weeks after cataract surgery, but it has occurred as early as 3 weeks or as late as 6 months postoperatively. ${ }^{99}$ The breakdown of the inner blood-retinal barrier causes the CME. The accumulation is centered around the foveola, with loss of the foveal depression. The diagnosis of CME can generally be made on the basis of a clinical examination with evidence of perifoveal cystic spaces and can be confirmed with use of FA to document the classic petaloid pattern of leakage, mainly into the outer retina. However OCT is more specific because it allows quantitative measurement of all the structures of the macular area.

OCT images demonstrate the cystic areas of decreased reflectivity within the neurosensory retina, consistent with the known histopathology of this entity (Figure 49). One clinical finding that does appear to correlate with visual acuity is the level of macular thickening. Therefore, there may be a role for the OCT in the diagnosis and monitoring of CME. Pseudophakic CME has cystic spaces that are visible as rounded low-scattering areas, which typically occur in the neurosensorial retina (low-reflective intraretinal areas) (Figure 50). The retinal thickness is easily measured between the nerve fiber layer and the highly backscattering red layer that represents the RPE/choriocapillaris complex. Again, the ability to quantify the extent of thickening is useful, not only in the diagnosis of this disease, but also, in assessing the response to treatment (topical medications, periocular, intraocular injections, and vitrectomy) (Figure 51).

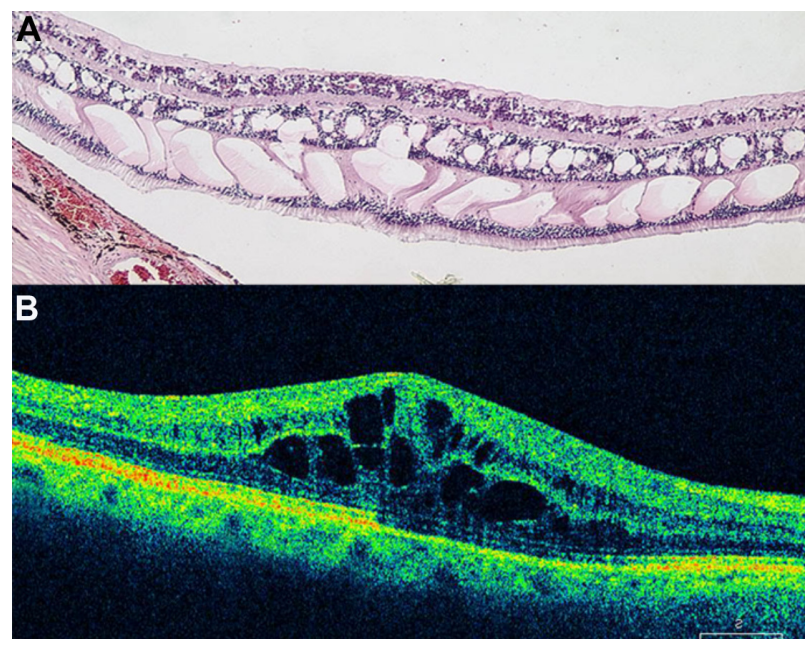

Figure 49 (A) Histological cross section of CME. (B) SD-OCT cross section of pseudophakic CME with retinal thickening, cystic intraretinal, and subretinal fluid. Note: Cirrus ${ }^{\mathrm{TM}}$ OCT allows precise characterization of the lesions, which previously could only be appreciated on histologic sections.

Abbreviations: CME, cystoid macular edema; OCT, optical coherence tomography; $\mathrm{SD}$, spectral domain.

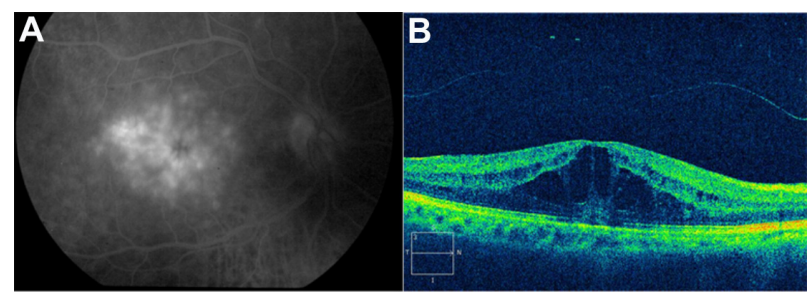

Figure 50 (A) Fluorescein angiography of CME in the recirculation phase of the angiogram demonstrates a classic petaloid leakage pattern of pseudophakic CME. (B) Cirrus ${ }^{\mathrm{TM}}$ optical coherence tomography demonstrates cystic macular changes as low-reflective spaces (dark spaces), more prominently at the level of the outer retinal layers.

Abbreviation: CME, cystoid macular edema.

\section{Idiopathic parafoveal telangiectasis}

Retinal telangiectasis is a developmental retinal vascular disorder characterized by an ectasia of capillaries of the retina, in which irregular capillary dilation and incompetence occur in the retinal periphery of the macula. Gass described a classification with three subgroups. ${ }^{100}$

OCT clearly depicts the involvement of the intraretinal and subretinal spaces in this condition. Parafoveal telangiectasis may show low-reflective intraretinal areas of macular edema and highly reflective lipid exudates on OCT (Figures 52 and 53). Plaques of RPE hyperplasia appear as intraretinal hyperreflective spots associated with shadowing of the reflections from the tissue below. In addition, OCT may show subretinal neovascularization associated with juxtafoveal telangiectasis. ${ }^{101}$ 




Figure 5 I Sequential Stratus OCT images from a 64-year-old man with a 4-month history of loss of vision, to 20/I60, in his right eye, in whom refractory pseudophakic cystoid macular edema had developed.

Notes: (A) Horizontal OCT scan obtained through the fovea revealing a loss of the normal foveal contour, diffuse macular thickening, and areas of low intraretinal reflectivity consistent with intraretinal cysts and fluid accumulation. The retinal map analysis revealed a foveal thickness of $595 \mu \mathrm{m}$. The patient underwent an intravitreal injection of bevacizumab at a dose of $2.5 \mathrm{mg}$ in this eye. (B) OCT scans revealing complete resolution of the cystic spaces and with restoration of foveal anatomic features at I month after the bevacizumab injection. The retinal map analysis indicates a central foveal thickness of $260 \mu \mathrm{m}$. VA improved to 20/63. (C) Six months after the injection, the OCT scan showed marked improvement in foveal thickness $(229 \mu \mathrm{m})$ and contour. The VA was 20/32. (D) OCT scans showing a normal-appearing macula at 12 months after injection. The foveal thickness decreased to $202 \mu \mathrm{m}$, and VA was 20/32. Reprinted with permission form Arevalo JF, Maia M, Garcia-Amaris RA, et al; Pan-American Collaborative Retina Study Group. Intravitreal bevacizumab for refractory pseudophakic cystoid macular edema: the Pan-American Collaborative Retina Study Group results. Ophthalmology. 2009; I 16(8):148|-1487. Copyright 2009 Elsevier. ${ }^{108}$

Abbreviations: OCT, optical coherence tomography; VA, visual acuity.

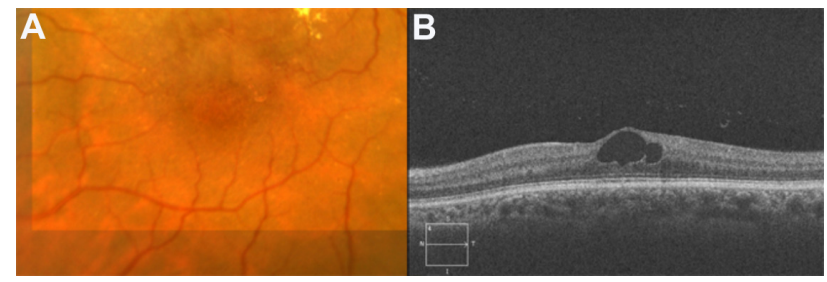

Figure 52 (A) Color fundus photograph of localized parafoveal telangiectasis. (B) Cirrus ${ }^{\mathrm{TM}}$ optical coherence tomography image shows an intraretinal cyst as a low-reflective intraretinal area, secondary to the parafoveal telangiectasis.

\section{Miscellaneous retinal diseases}

OCT is also useful for analyzing other retinal diseases, such as hydroxychloroquine toxicity (Figure 54), radiation retinopathy (Figure 55), commotio retinae (Figure 56), retinochoroidal coloboma (Figure 57), foveal hypoplasia in albinism (Figure 58), medullary aplasia (Figure 59), ruptured macroaneurysm (Figures 60 and 61), Coats' disease
(Figure 62), and to confirm the presence of subretinal liquid perfluorocarbon after surgery (Figure 63).

\section{Enhanced depth imaging}

The posterior ciliary artery circulation is the main source of blood supply for the choroid, the AMD, and the outer retina. ${ }^{102}$ Contact $\mathrm{B}$-scan ultrasonography can provide evidence of thickening in the disease state, and indocyanine green angiography is helpful in examining vascular structures in the choroid, but neither supplies accurate cross-sectional information. Conventional SD-OCT devices have difficulty with imaging the choroid because of the decreasing sensitivity and resolution with increasing displacement from zero-delay, the decreased maximal dynamic range inherent in SD systems, wavelength-dependent light scattering and signal loss in the image path, and the lateral width of the defocused imaging beam. ${ }^{85}$ 

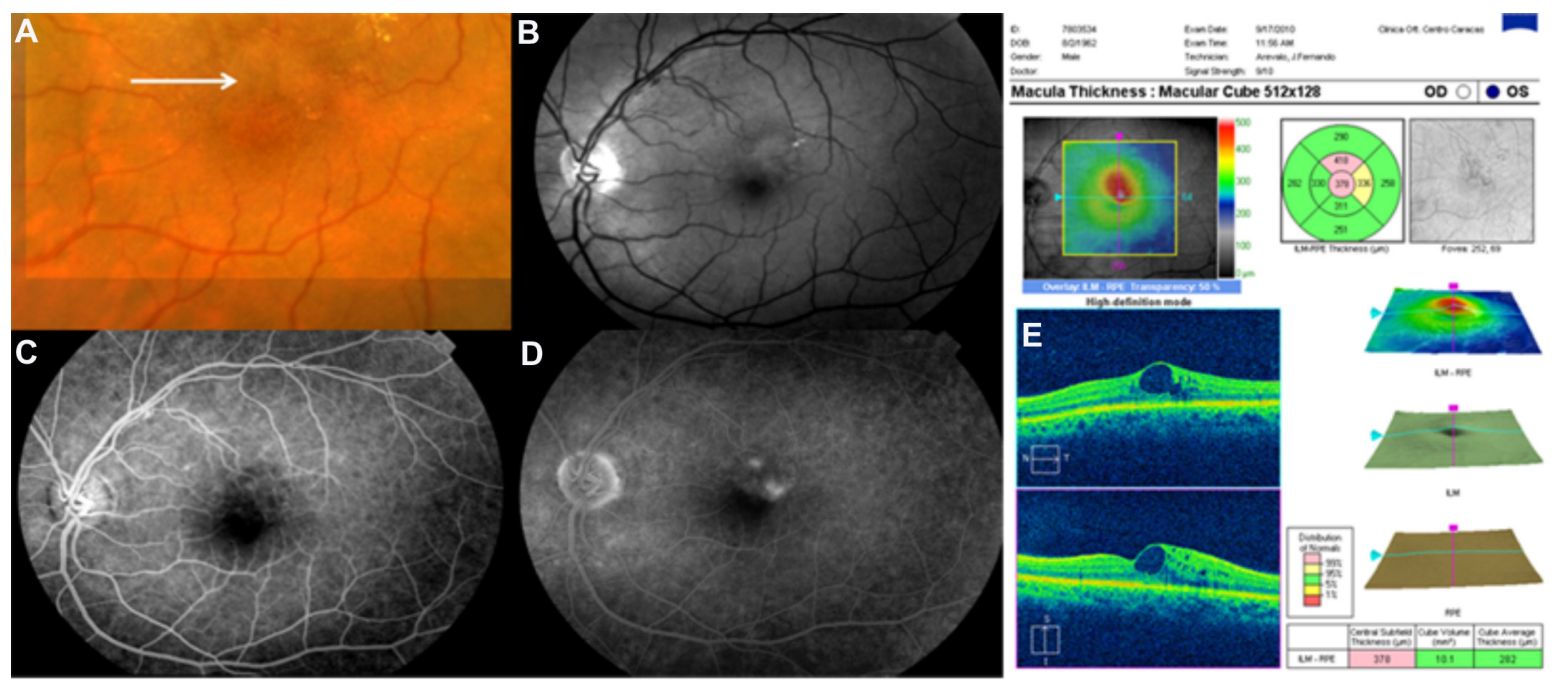

Figure 53 (A) Color fundus photograph of localized parafoveal telangiectasis. (B-D) Fluorescein angiography shows hyperfluorescence (D) due to late extravasation of the fluorescein dye. (E) The Cirrus ${ }^{\mathrm{TM}}$ optical coherence tomography image shows a intraretinal cyst as a low-reflective intraretinal area, secondary to parafoveal telangiectasis.

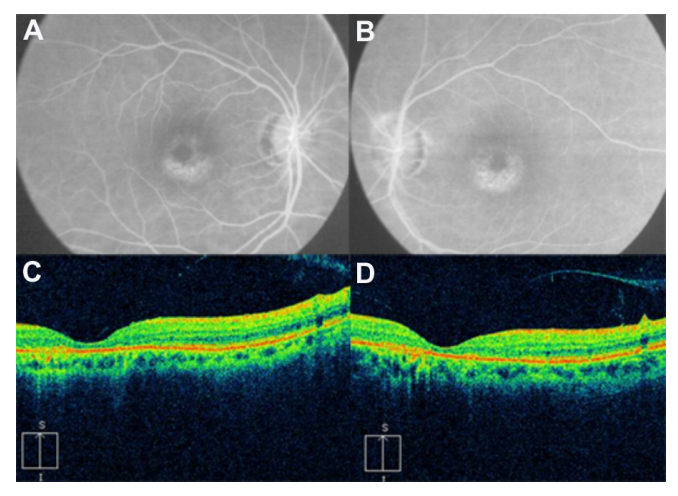

Figure 54 Fluorescein angiograms of the right $(\mathbf{A})$ and left $(\mathbf{B})$ eyes of a patient with hydroxychloroquine maculopathy. (C-D) Spectral domain Cirrus ${ }^{\mathrm{TM}}$ optical coherence tomography scans of the same two eyes.

Notes: Lack of retinal pigment through to the choroidal circulation is shown as a ringlike retinal depigmentation in the angiograms. The Spectral domain Cirrus ${ }^{\mathrm{TM}}$ optical coherence tomography shows significant thinning of the outer retinal structures in the foveal region of both the right (C) and left (D) eyes.

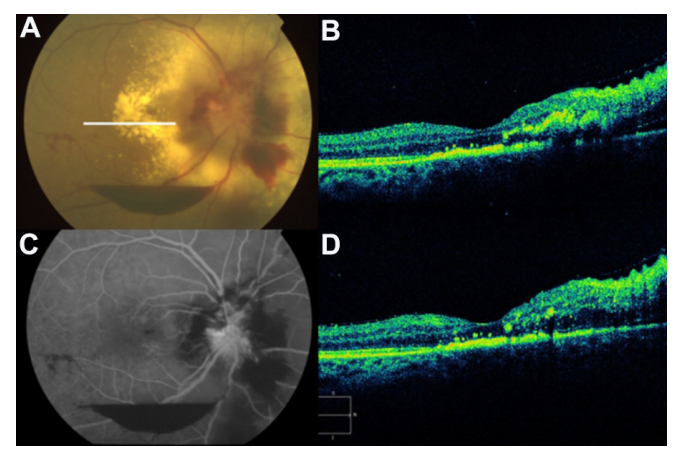

Figure 55 (A and $\mathbf{C}$ ) Color fundus photograph and fluorescein angiogram of the right eye demonstrate papilledema, subhyaloid and retinal hemorrhages, and extensive peripapillary lipid exudation in the posterior pole, secondary to radiation retinopathy. (B and D) Spectral domain optical Cirrus ${ }^{\mathrm{TM}}$ coherence tomography image of the same eye, with noticeably increased nasal retinal thickness and hard exudates in the inner nuclear layer, outer plexiform layer, and outer nuclear layer.

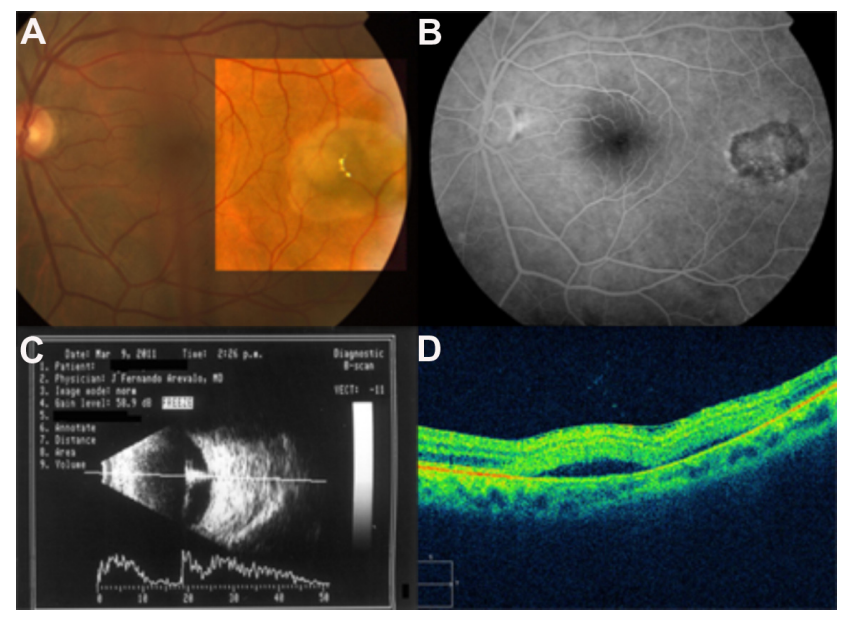

Figure 56 (A) Color fundus photograph, (B) fluorescein angiogram, (C) B-scan ultrasound, and (D) Cirrus ${ }^{\mathrm{TM}}$ optical coherence tomography image demonstrate commotio retinae and an intraocular foreign body.

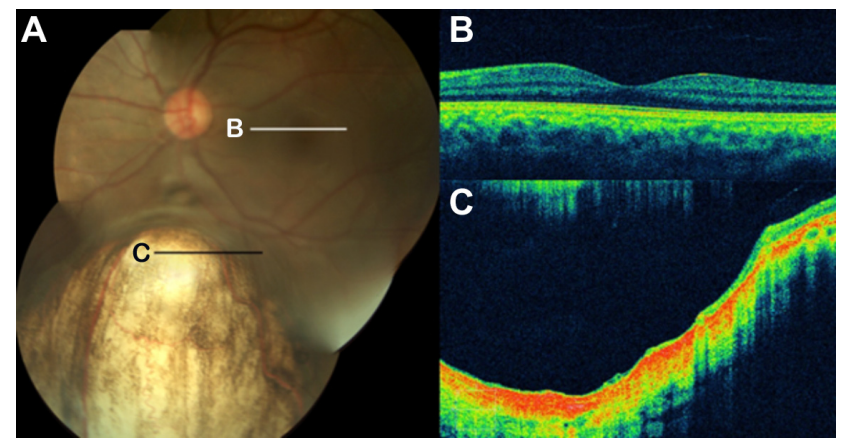

Figure 57 (A) Color fundus photograph of a retinochoroidal coloboma. (B) Normal foveal contour. (C) Cirrus ${ }^{\mathrm{TM}}$ optical coherence tomography shows the typical features of retinochoroidal coloboma with the lack of retinal and choroidal tissues, and light backscatter.

Note: The black and white lines depict the direction of the line scan. 


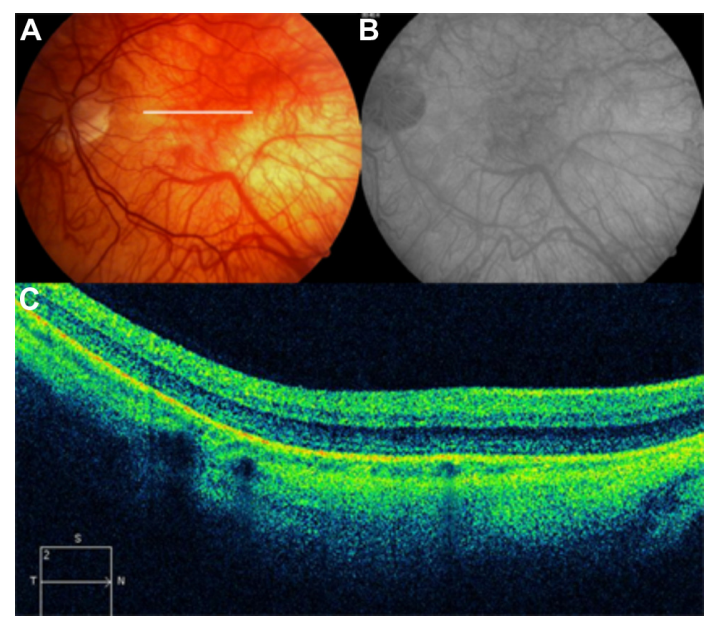

Figure 58 Foveal hypoplasia in albinism.

Notes: (A) Fundus color and (B) red-free photographs of the left eye show a clear view of choroidal vasculature due to the hypopigmentation of the retinal pigment epithelium, a pale retina, foveal hypoplasia, and an indistinct optic disc margin (C) Cirrus ${ }^{\mathrm{TM}}$ optical coherence tomography image of the same eye, showing the absence of the normal macular depression and high reflectivity across the fovea. The white line depicts the direction of the OCT scan.

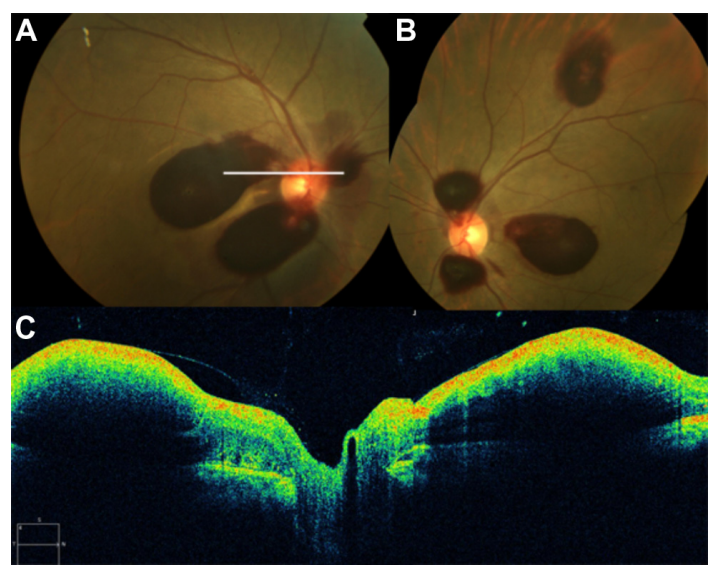

Figure 59 (A and B) Fundus color photographs of both eyes show scattered preretinal hemorrhages at the macula and surrounding the optic disk, secondary to medullary aplasia. (C) The Cirrus ${ }^{\mathrm{TM}}$ OCT image shows increased thickness and reflectivity due to the presence of blood over the inner retinal layers, causing shadowing of the optical signals of the outer retinal layers and the retinal pigment epithelium/choriocapillaris complex.

Note: The white line demonstrates the direction of the OCT scan.

Abbreviation: OCT, optical coherence tomography.

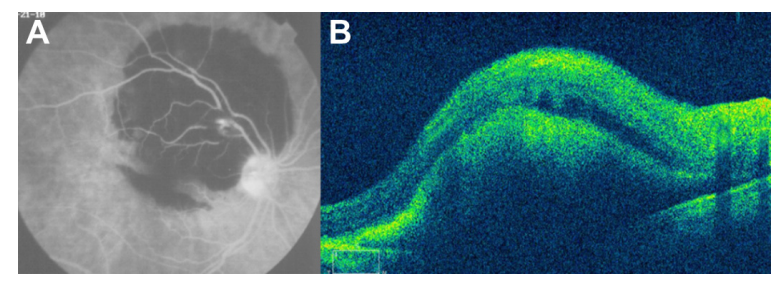

Figure 60 (A) Fluorescein angiography shows leakage from the aneurysm and surrounding area that is blocked by preretinal and retinal hemorrhage. (B) Spectraldomain Cirrus ${ }^{\mathrm{TM}}$ optical coherence tomography showing an active retinal arterial macroaneurysm.

Notes: Increased central retinal thickness and subfoveal elevation of the sensory retina is seen in $(\mathbf{B})$.

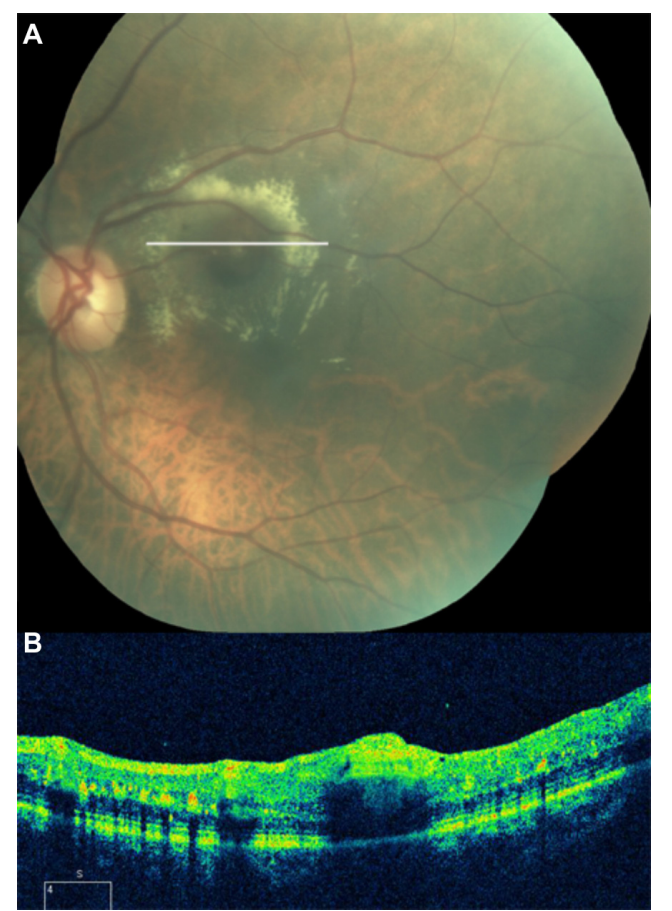

Figure 6I (A) Fundus color photograph shows retinal arterial macroaneurysm and surrounding lipid deposits I month after presentation. (B) Cirrus ${ }^{\mathrm{TM}}$ optical coherence tomography image shows the retinal arterial macroaneurysm located in the superficial layers and accompanied by multiple lipid deposits, predominantly seen in the outer plexiform layer.

Note: The white line depicts the direction of the OCT scan.

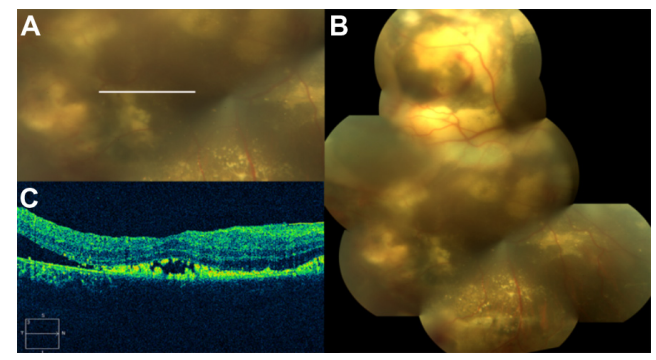

Figure 62 Coats' disease.

Notes: (A and B) Fundus color photograph, at presentation, shows subretinal lipid exudates extending posteriorly and exudative retinal detachment in the right eye. (C) The Cirrus ${ }^{\mathrm{TM}}$ optical coherence tomography image shows neurosensorial retinal detachment, intraretinal fluid, and multiple lipid exudates in the outer retinal layer. The white line depicts the direction of the OCT scan.

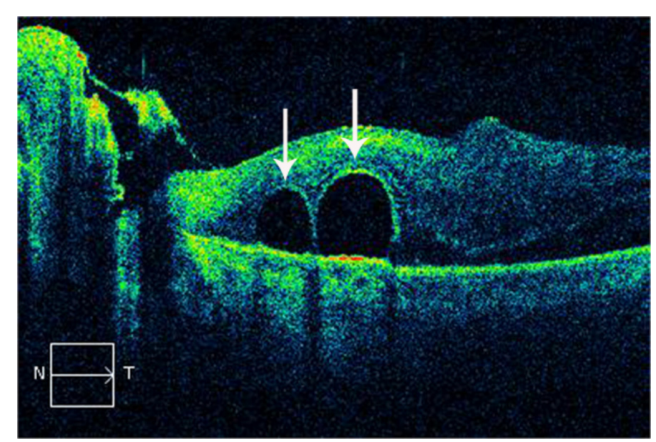

Figure 63 Spectral-domain Cirrus ${ }^{\mathrm{TM}}$ optical coherence tomography image demonstrates the presence of two subretinal bubbles of perfluorocarbon liquid (white arrows) after vitreoretinal surgery. 
The newly developed EDI SD-OCT enables in vivo cross-sectional imaging of the choroid. ${ }^{85}$ EDI-OCT is a simple modification of the SD-OCT technique. EDI OCT uses SD-OCT that is positioned closer to the eye than usual, such that a stable inverted image is produced. The net effect of this practice is that the sensitivity of the imaging in the deeper layers of tissue is increased (Figures 64 and 65). ${ }^{85} \mathrm{In}$ this fashion, EDI OCT may represent a useful approach to in vivo investigation of the choroidal changes in eyes, with several macular diseases.

Using EDI OCT in a group of healthy subjects, Margolis and Spaide ${ }^{86}$ found a mean subfoveal choroidal thickness of $287 \mu \mathrm{m}$, with a negative correlation with age. By linear regression, they estimated the reduction to be approximately $15 \mu \mathrm{m}$ for each decade of life. ${ }^{86}$ In highly myopic eyes, a reduced choroidal thickness (mean: $93 \mu \mathrm{m}$ ), with a negative correlation with refractive error, was found. ${ }^{103}$ The images acquired with EDI OCT have improved the ability to visualize the choroid and its internal architecture. The relative lack of pigment in patients with blond fundus and the EDI OCT technology may allow an extremely clear visualization of the choroid and sclera, showing the intrascleral course and the penetration of posterior ciliary arteries into the choroid. ${ }^{104}$

EDI OCT was used to examine retinal PED in AMD to learn more about the internal structure of these conditions. ${ }^{59}$ Then, Querques et al ${ }^{105}$ investigated the changes in macular choroidal thickness in eyes with various stages of diabetic retinopathy, using this technology. They noted that in diabetic eyes, there was an overall thinning of the choroid on EDI OCT. The mean subfoveal choroidal thickness was significantly reduced in the diabetic group compared with the control group $(P<0.001)$. They concluded that a decreased choroidal thickness may lead to tissue hypoxia and consequently increase the level of VEGF, resulting in the breakdown of the blood-retinal barrier and the development of macular edema. ${ }^{105}$

Reibaldi et a ${ }^{106}$ observed the choroidal thickness in the macular area in patients with idiopathic macular hole in one eye and an unaffected fellow eye, and in healthy controls. The choroidal thickness was reduced in the eyes with idiopathic macular hole and also in the fellow unaffected eyes. This may suggest a contributing role of the choroid in the pathogenesis of idiopathic macular hole. ${ }^{106}$ Choroidal thickness varies according to the location in relation to the macula. The inferior macular choroid is thinner than the superior macular choroid, and the choroid is thinnest nasally, thickest subfoveally, and thinner temporally. Using EDI OCT, choroidal thickness was studied in normal eyes and was shown to be thickest underneath the fovea and to be inversely proportional to age. ${ }^{86}$

Recently, Coscas et al ${ }^{107}$ reported a method developed to visualize and localize the neovascular network in the internal

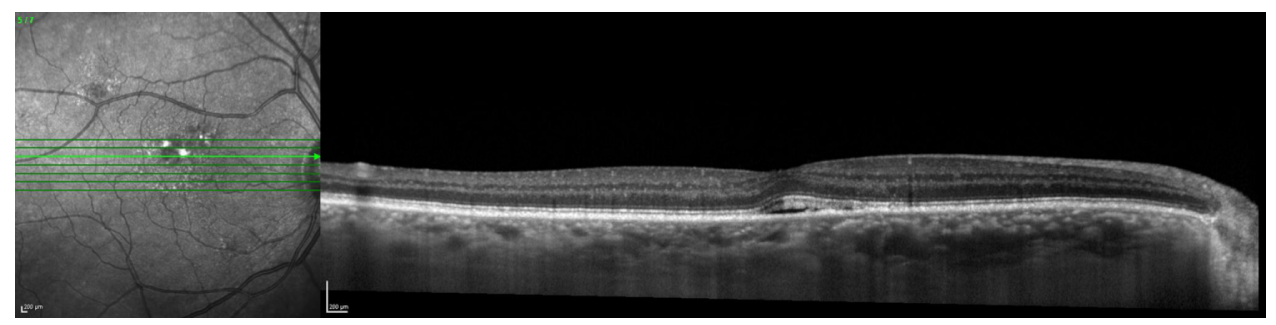

Figure 64 Spectralis ${ }^{\circledR}$ optical coherence tomography image (Courtesy of Roberto Gallego-Pinazo, MD).

Notes: Foveal-centered EDI OCT image of a 72-year-old Caucasian male with a 26-year history of central serous chorioretinopathy and with a present acute episode of serous foveal detachment. The subfoveal choroidal thickness measured 437 microns.

Abbreviation: EDI OCT, enhanced depth imaging optical coherence tomography.

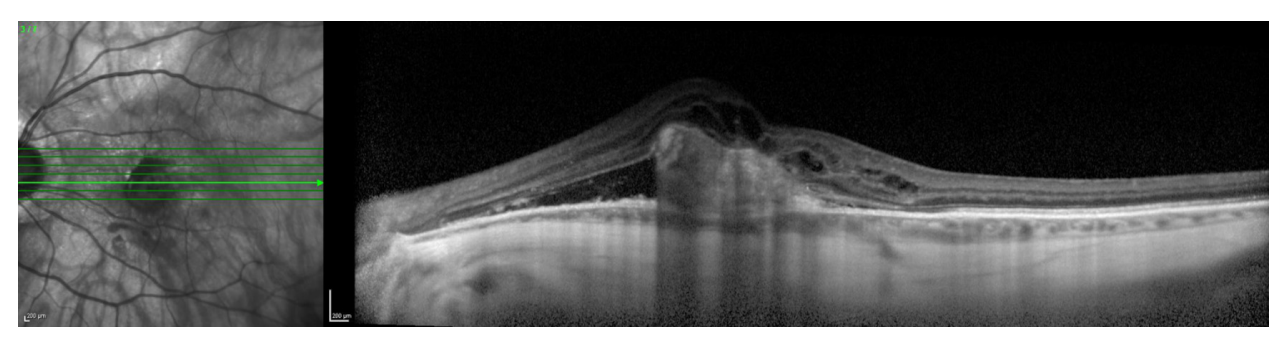

Figure 65 Spectralis ${ }^{\circledR}$ optical coherence tomography image (Courtesy of Roberto Gallego-Pinazo, MD).

Notes: Foveal-centered EDI OCT image of a 63 -year-old highly myopic Caucasian male who developed type 2 neovascularization. The subfoveal choroidal thickness measured 136 microns.

Abbreviation: EDI OCT, enhanced depth imaging optical coherence tomography. 
structure of fibrovascular PED due to AMD, using the en face EDI-OCT technique. This analysis may offer a clearer picture of the neovascular process and provide insights into the pathophysiology of fibrovascular PED development. They concluded that the en face EDI-OCT technique enables visualization and localization of the entire branching neovascular network of CNV within fibrovascular PED, without the need for dye injection. ${ }^{107}$

\section{Summary}

OCT is a noninvasive technique that can also directly analyze the impact of CNV in AMD on the outer retinal layers and can evaluate alterations of the photoreceptors and inner and outer segments. OCT can help establish treatment and retreatment guidelines and help to monitor treatment failure or success. OCT facilitates the quantification of the retinal thickness and allows precise evaluation, in an objective way, of the effect of the management of diabetic patients with different degrees of diabetic retinopathy and clinically significant macular edema. In addition, we can use this technology to evaluate specific features that may serve as predictive factors in the prognosis and follow up of the most common posterior pole pathologies.

With SD-OCT, advantages such as improved retinal coverage, registration of OCT findings to fundus features, longitudinal tracking of pathology, and 3D imaging, promise to improve the sensitivity and specificity of diagnosis and to enhance our understanding of disease pathogenesis. SD-OCT scans of the outer retinal layers provide new morphological, qualitative, and quantitative data that are valuable for diagnosis, for developing the best treatment strategy, and for monitoring treatment. Earlier markers of disease may be detected, possibly identifying different candidates for medical or surgical therapies.

SD-OCT has already proven to be invaluable, and it should be part of our armamentarium along with slit lamp biomicroscopy and FA. In addition, in the near future, we predict this useful tool will be widely available in the operating room, to support surgical decisions and to monitor immediate results.

\section{Acknowledgment}

This work was supported in part by the Arevalo-Coutinho Foundation for Research in Ophthalmology (FACO), Caracas, Venezuela. Presented in part at the LIX National Congress of Ophthalmology, Caracas, Venezuela, May 2011.

\section{Disclosure}

The authors report no conflicts of interest in this work.

\section{References}

1. Otani T, Kishi S, Maruyama Y. Patterns of diabetic macular edema with optical coherence tomography. Am J Ophthalmol. 1999;127(6): 688-693.

2. Mylonas G, Bolz M, Kriechbaum K, et al. Retinal architecture recovery after grid photocoagulation in diabetic macular edema observed in vivo by spectral domain optical coherence tomography. Retina. 2013;33(4):717-725.

3. Martidis A, Duker JS, Greenberg PB, et al. Intravitreal triamcinolone for refractory diabetic macular edema. Ophthalmology. 2002;109(5):920-927.

4. Jonas JB, Kreissig I, Söfker A, Degenring RF. Intravitreal injection of triamcinolone for diffuse diabetic macular edema. Arch Ophthalmol. 2003;121(1):57-61.

5. Hannouche RZ, Avila MP, Isaac DL, Silva RS, Rassi AR. Correlation between central subfield thickness, visual acuity and structural changes in diabetic macular edema. Arq Bras Oftalmol. 2012;75(3):183-187.

6. Shimura M, Yasuda K, Yasuda M, Nakazawa T. Visual outcome after intravitreal bevacizumab depends on the optical coherence tomographic patterns of patients with diffuse diabetic macular edema. Retina. 2013;33(4):740-747.

7. Ito S, Miyamoto N, Ishida K, Kurimoto Y. Association between external limiting membrane status and visual acuity in diabetic macular oedema. Br J Ophthalmol. 2013;97(2):228-232.

8. Klein R, Klein BE, Linton KL. Prevalence of age-related maculopathy: The Beaver Dam Eye Study. Ophthalmology. 1992;99(6):933-943.

9. Krebs I, Brannath W, Glittenberg C, Zeiler F, Sebag J, Binder S. Posterior vitreomacular adhesion: a potential risk factor for exudative age-related macular degeneration? Am J Ophthalmol. 2007;144(5):741-746.

10. Robison CD, Krebs I, Binder S, et al. Vitreomacular adhesion in active and end-stage age-related macular degeneration. Am J Ophthalmol. 2009;148(1):79-82. e2.

11. Yi K, Mujat M, Park BH, et al. Spectral domain optical coherence tomography for quantitative evaluation of drusen and associated structural changes in non-neovascular age-related macular degeneration. $\mathrm{Br}$ J Ophthalmol. 2009;93(2):176-181.

12. Hee MR, Baumal CR, Puliafito CA, et al. Optical coherence tomography of age-related macular degeneration and choroidal neovascularization. Ophthalmology. 1996;103(8):1260-1270.

13. Friedman SM, Margo CE. Choroidal neovascular membranes: reproducibility of angiographic interpretation. Am J Ophthalmol. 2000;130(6): 839-841.

14. van Velthoven ME, de Smet MD, Schlingemann RO, Magnani M, Verbraak FD. Added value of OCT in evaluating the presence of leakage in patients with age-related macular degeneration treated with PDT. Graefes Arch Clin Exp Ophthalmol. 2006;244(9):1119-1123.

15. Fung AE, Lalwani GA, Rosenfeld PJ, et al. An optical coherence tomography-guided, variable dosing regimen with intravitreal ranibizumab (Lucentis) for neovascular age-related macular degeneration. Am J Ophthalmol. 2007;143(4):566-583.

16. Zhang N, Hoffmeyer GC, Young ES, et al. Optical coherence tomography reader agreement in neovascular age-related macular degeneration. Am J Ophthalmol. 2007;144(1):37-44.

17. Krebs I, Ansari-Shahrezaei S, Goll A, Binder S. Activity of neovascular lesions treated with bevacizumab: comparison between optical coherence tomography and fluorescein angiography. Graefes Arch Clin Exp Ophthalmol. 2008;246(6):811-815.

18. Malamos P, Sacu S, Georgopoulos M, Kiss C, Pruente C, SchmidtErfurth U. Correlation of high-definition optical coherence tomography and fluorescein angiography imaging in neovascular macular degeneration. Invest Ophthalmol Vis Sci. 2009;50(10):4926-4933.

19. Chong EW, Wong TY, Kreis AJ, Simpson JA, Guymer RH. Dietary antioxidants and primary prevention of age related macular degeneration: systematic review and meta-analysis. BMJ. 2007;335(7623):755.

20. Age-Related Eye Disease Study Research Group. A randomized, placebocontrolled, clinical trial of high-dose supplementation with vitamins $\mathrm{C}$ and $\mathrm{E}$, beta-carotene, and zinc for age-related macular degeneration and vision loss: AREDS report no. 8. Arch Ophthalmol. 2001;119(10):1417-1436. 
21. Gass JD. Drusen and disciform macular detachment and degeneration. Arch Ophthalmol. 1973;90(3):206-217.

22. Davis MD, Gangnon RE, Lee LY, et al; Age-Related Eye Disease Study Group. The Age-Related Eye Disease Study severity scale for agerelated macular degeneration: AREDS report no 17. Arch Ophthalmol. 2005;123(11):1484-1498

23. Ferris FL, Davis MD, Clemons TE, et al; Age-Related Eye Disease Study (AREDS) Research Group. A simplified severity scale for agerelated macular degeneration: AREDS report no 18. Arch Ophthalmol. 2005;123(11):1570-1574.

24. Klein R, Klein BE, Knudtson MD, Meuer SM, Swift M, Gangnon RE. Fifteen-year cumulative incidence of age-related macular degeneration: the Beaver Dam Eye Study. Ophthalmology. 2007;114(2):253-262.

25. Klein R, Peto T, Bird A, Vannewkirk MR. The epidemiology of age-related macular degeneration. Am J Ophthalmol. 2004;137(3): 486-495.

26. Sarks SH. Drusen patterns predisposing to geographic atrophy of the retinal pigment epithelium. Aust J Ophthalmol. 1982;10(2):91-97.

27. Sebag M, Peli E, Lahav M. Image analysis of changes in drusen area. Acta Ophthalmol (Copenh). 1991;69(5):603-610.

28. Wang JJ, Foran S, Smith W, Mitchell P. Risk of age-related macular degeneration in eyes with macular drusen or hyperpigmentation: the Blue Mountains Eye Study cohort. Arch Ophthalmol. 2003;121(5): 658-663.

29. Green WR, Enger C. Age-related macular degeneration histopathologic studies. The 1992 Lorenz E. Zimmerman Lecture. Retina. 1993;100(10):1519-1535.

30. Klein R, Klein BE, Tomany SC, Meuer SM, Huang GH. Ten-year incidence and progression of age-related maculopathy: The Beaver Dam eye study. Ophthalmology. 2002;109(10):1767-1779.

31. Bressler NM, Munoz B, Maguire MG, et al. Five-year incidence and disappearance of drusen and retinal pigment epithelial abnormalities. Waterman study. Arch Ophthalmol. 1995;113(3):301-308.

32. Casswell AG, Kohen D, Bird AC. Retinal pigment epithelial detachments in the elderly: classification and outcome. $\mathrm{Br} J$ Ophthalmol. 1985;69(6):397-403.

33. Hartnett ME, Weiter JJ, Garsd A, Jalkh AE. Classification of retinal pigment epithelial detachments associated with drusen. Graefes Arch Clin Exp Ophthalmol. 1992;230(1):11-19.

34. Pauleikhoff D, Löffert D, Spital G, et al. Pigment epithelial detachment in the elderly. Clinical differentiation, natural course and pathogenetic implications. Graefes Arch Clin Exp Ophthalmol. 2002;240(7):533-538.

35. Coscas F, Coscas G, Souied E, Tick S, Soubrane G. Optical coherence tomography identification of occult choroidal neovascularization in age-related macular degeneration. Am J Ophthalmol. 2007;144(4):592-599.

36. Age-Related Eye Disease Study Research Group. The Age-Related Eye Disease Study system for classifying age-related macular degeneration from stereoscopic color fundus photographs: the AgeRelated Eye Disease Study Report Number 6. Am J Ophthalmol. 2001;132(5):668-681.

37. Spraul CW, Grossniklaus HE. Characteristics of Drusen and Bruch's membrane in postmortem eyes with age-related macular degeneration. Arch Ophthalmol. 1997;115(2):267-273.

38. Roquet W, Roudot-Thoraval F, Coscas G, Soubrane G. Clinical features of drusenoid pigment epithelial detachment in age related macular degeneration. Br J Ophthalmol. 2004;88(5):638-642.

39. Cukras C, Agrón E, Klein ML, et al; Age-Related Eye Disease Study Research Group. Natural history of drusenoid pigment epithelial detachment in age-related macular degeneration: age-related eye disease study report no 28. Ophthalmology. 2010;117(3):489-499.

40. Querques G, Bux AV, Delle Noci N. Foveal geographic atrophy following intravitreal pegaptanib sodium (Macugen) for drusenoid pigment epithelium detachment. Eur J Ophthalmol. 2009;19(5):890-893.
41. Krishnan R, Lochhead J. Regression of soft drusen and drusenoid pigment epithelial detachment following intravitreal anti-vascular endothelial growth factor therapy. Can J Ophthalmol. 2010;45(1):83-84.

42. Gallego-Pinazo R, Marina A, Suelves-Cogollos, et al. Intravitreal ranibizumab for symptomatic drusenoid pigment epithelial detachment without choroidal neovascularization in age-related macular degeneration. Clin Ophthalmol. 2011;5:161-165.

43. Yannuzzi LA, Hope-Ross M, Slakter JS, et al. Analysis of vascularized pigment epithelial detachments using indocyanine green videoangiography. Retina. 1994;14(2):99-113.

44. Cho M, Athanikar A, Paccione J, Wald KJ. Optical coherence tomography features of acute central serous chorioretinopathy versus neovascular age-related macular degeneration. $\mathrm{Br} J$ Ophthalmol. 2010;94(5):597-599.

45. Bressler SB, Silva JC, Bressler NM, Alexander J, Green WR. Clinicopathologic correlation of occult choroidal neovascularization in age-related macular degeneration. Arch Ophthalmol. 1992;110(6):827-832.

46. Ting TD, Oh M, Cox TA, Meyer CH, Toth CA. Decreased visual acuity associated with cystoid macular edema in neovascular age-related macular degeneration. Arch Ophthalmol. 2002;120(6):731-737.

47. Ozawa S, Ishikawa $\mathrm{K}$, Ito $\mathrm{Y}$, et al. Differences in macular morphology between polypoidal choroidal vasculopathy and exudative age-related macular degeneration detected by optical coherence tomography. Retina. 2009;29(6):793-802.

48. Lambert HM, Capone A Jr, Aaberg TM, Sternberg P Jr, Mandell BA, Lopez PF. Surgical excision of subfoveal neovascular membranes in age-related macular degeneration. Am J Ophthalmol. 1992;113(3): 257-262.

49. Weber-Krause B, Eckardt U. Incidence of posterior vitreous detachment in eyes with and without age-related macular degeneration. An ultrasonic study. Ophthalmologe. 1996;93(6):660-665. German.

50. Ondeş F, Yilmaz G, Acar MA, Unlü N, Kocaoğlan H, Arsan AK. Role of the vitreous in age-related macular degeneration. Jpn J Ophthalmol. 2000;44(1):91-93.

51. Mojana F, Cheng L, Bartsch DU, et al. The role of abnormal vitreomacular adhesion in age-related macular degeneration: spectral optical coherence tomography and surgical results. Am J Ophthalmol. 2008;146(2):218-227.

52. Quaranta-El Maftouhi M, Mauget-Faÿsse M. Anomalous vitreoretinal adhesions in patients with exudative age-related macular degeneration: an OCT study. Eur J Ophthalmol. 2006;16(1):134-137.

53. Toth CA, Birngruber R, Boppart SA, et al. Argon laser retinal lesions evaluated in vivo by optical coherence tomography. Am J Ophthalmol. 1997;123(2):188-198.

54. Rosenfeld PJ, Brown DM, Heier JS, et al; MARINA Study Group. Ranibizumab for neovascular age-related macular degeneration. $N$ Engl J Med. 2006;355(14):1419-1431.

55. Kaiser PK, Brown DM, Zhang K, et al. Ranibizumab for predominantly classic neovascular age-related macular degeneration: subgroup analysis of first-year ANCHOR results. Am J Ophthalmol. 2007;144(6): 850-857.

56. Krebs I, Hagen S, Brannath W, et al. Repeatability and reproducibility of retinal thickness measurements by optical coherence tomography in age-related macular degeneration. Ophthalmology. 2010;117(8): $1577-1584$

57. Sayanagi K, Sharma S, Yamamoto T, Kaiser PK. Comparison of spectral-domain versus time-domain optical coherence tomography in management of age-related macular degeneration with ranibizumab. Ophthalmology. 2009;116(5):947-955.

58. Querques G, Forte R, Berboucha E, et al. Spectral-domain versus time domain optical coherence tomography before and after ranibizumab for agerelated macular degeneration. Ophthalmic Res. 2011;46(3): 152-159.

59. Spaide RF. Enhanced depth imaging optical coherence tomography of retinal pigment epithelial detachment in age-related macular degeneration. Am J Ophthalmol. 2009;147(4):644-652. 
60. Early Treatment Diabetic Retinopathy Study Research Group. Photocoagulation for diabetic macular edema. Early Treatment Diabetic Retinopathy Study report number 1. Arch Ophthalmol. 1985;103(12): 1796-1806.

61. Moss SE, Klein R, Klein BE. The 14-year incidence of visual loss in a diabetic population. Ophthalmology. 1998;105(6):998-1003.

62. Klein R, Klein BE, Moss SE, Davis MD, DeMets DL. The Wisconsin epidemiologic study of diabetic retinopathy. IV. Diabetic macular edema. Ophthalmology. 1984;91(12):1464-1474.

63. Gieser JP, Rusin MM, Mori M, Blair NP, Shahidi M. Clinical assessment of the macula by retinal topography and thickness mapping. $\mathrm{Am}$ J Ophthalmol. 1997;124(5):648-660.

64. Baumann M, Gentile RC, Liebmann JM, Ritch R. Reproducibility of retinal thickness measurements in normal eyes using optical coherence tomography. Ophthalmic Surg Lasers. 1998;29(4):280-285.

65. Koozekanani D, Roberts C, Katz SE, Herderick EE. Intersession repeatability of macular thickness measurements with the Humphrey 2000 OCT. Invest Ophthalmol Vis Sci. 2000;41(6):1486-1491.

66. Yanoff M, Fine BS, Brucker AJ, Eagle RC. Pathology of human cystoid macular edema. Surv Ophthalmol. 1984;28 Suppl:S505-S511.

67. Fine BS, Brucker AJ. Macular edema and cystoid macular edema. Am J Ophthalmol. 1981;92(4):466-481.

68. Tso MO. Pathology of cystoid macular edema. Ophthalmology. 1982; 89(8):902-915.

69. Schuman JS, Pedut-Kloizman T, Hertzmark E, et al. Reproducibility of nerve fiber layer thickness measurements using optical coherence tomography. Ophthalmology. 1996;103(11):1889-1898.

70. Punjabi OS, Flynn HW Jr, Knighton RW, et al. Spectral domain optical coherence tomography for proliferative diabetic retinopathy with subhyaloid hemorrhage. Ophthalmic Surg Lasers Imaging. 2008;39(6): 494- 496.

71. Kroll P, Wiegand W, Schmidt J. Vitreopapillary traction in proliferative diabetic vitreoretinopathy [see comments]. $\mathrm{Br} J$ Ophthalmol. 1999;83(3):261-264

72. Kitaya N, Nagaoka T, Hikichi T, et al. Features of abnormal choroidal circulation in central serous chorioretinopathy. $\mathrm{Br} J$ Ophthalmol. 2003;87(6):709-712.

73. Montero JA, Ruiz-Moreno JM. Optical coherence tomography characterisation of idiopathic central serous chorioretinopathy. Br J Ophthalmol. 2005;89(5):562-564.

74. Gass JD. Pathogenesis of disciform detachment of the neuroepithelium Am J Ophthalmol. 1967;63(3):Suppl:S1-S139.

75. Spaide RF, Goldbaum M, Wong DW, Tang KC, Iida T. Serous detachment of the retina. Retina. 2003;23(6):820-846; quiz 895.

76. Guyer DR, Yannuzzi LA, Slakter JS, Sorenson JA, Ho A, Orlock D. Digital indocyanine green videoangiography of central serous chorioretinopathy. Arch Ophthalmol. 1994;112(8):1057-1062.

77. Spaide RF, Campeas L, Haas A, et al. Central serous chorioretinopathy in younger and older adults. Ophthalmology. 1996;103(12):2070-2079, discussion 2079-2080.

78. Iida T, Kishi S, Hagimura N, Shimizu K. Persistent and bilateral choroidal vascular abnormalities in central serous chorioretinopathy. Retina. 1999;19(6):508-512.

79. Prünte C, Flammer J. Choroidal capillary and venous congestion in central serous chorioretinopathy. Am J Ophthalmol. 1996;121(1):26-34.

80. Giovannini A, Scassellati-Sforzolini B, D'Altobrando E, Mariotti C, Rutili T, Tittarelli R. Choroidal findings in the course of idiopathic serous pigment epithelium detachment detected by indocyanine green videoangiography. Retina. 1997;17(4):286-293.

81. Hirami Y, Tsujikawa A, Sasahara M, et al. Alterations of retinal pigment epithelium in central serous chorioretinopathy. Clin Experiment Ophthalmol. 2007;35(3):225-230.

82. Fujimoto H, Gomi F, Wakabayashi T, Sawa M, Tsujikawa M, Tano Y. Morphologic changes in acute central serous chorioretinopathy evaluated by fourier-domain optical coherence tomography. Ophthalmology. 2008;115(9):1494-1500, 1500. e1.
83. Wang GH, Zhang J, Zhang D, Lv FL, Wang LX. Value of threedimensional optical coherence tomography and fundus photochromy in correlating the fluorescein leaking sites of acute central serous chorioretinopathy. Med Princ Pract. 2011;20(3):283-286.

84. Hee MR, Puliafito CA, Wong C, et al. Optical coherence tomography of central serous chorioretinopathy. Am J Ophthalmol. 1995;120(1): 65-74.

85. Spaide RF, Koizumi H, Pozzoni MC, Pozonni MC. Enhanced depth imaging spectral-domain optical coherence tomography. Am J Ophthalmol. 2008;146(4):496-500.

86. Margolis R, Spaide RF. A pilot study of enhanced depth imaging optical coherence tomography of the choroid in normal eyes. Am J Ophthalmol. 2009;147(5):811-815.

87. Imamura Y, Fujiwara T, Margolis R, Spaide RF. Enhanced depth imaging optical coherence tomography of the choroid in central serous chorioretinopathy. Retina. 2009;29(10):1469-1473.

88. Hussain D, Gass JD. Idiopathic central serous chorioretinopathy. Indian J Ophthalmol. 1998;46(3):131-137.

89. Schatz H, Madeira D, Johnson RN, McDonald HR. Central serous chorioretinopathy occurring in patients 60 years of age and older. Ophthalmology. 1992;99(1):63-67.

90. Wang M, Sander B, Lund-Anderson H, Larsen M. Detection of shallow detachments in central serous chorioretinopathy. Acta Ophthalmol Scand. 1999;77(4):402-405.

91. Yanoff M, Fine BS. Ocular Pathology. A Text and Atlas. 3rd ed. Hagerstown, MD: JB Lippincott Co; 1989.

92. Spaide RF, Lee JK, Klancnik JK Jr, Gross NE. Optical coherence tomography of branch retinal vein occlusion. Retina. 2003;23(3): 343-347.

93. Greenberg PB, Martidis A, Rogers AH, Duker JS, Reichel E. Intravitreal triamcinolone acetonide for macular oedema due to central retinal vein occlusion. Br J Ophthalmol. 2002;86(2):247-248.

94. Ichibe M, Yoshizawa T, Murakami K, et al. Surgical management of retinal detachment associated with myopic macular hole: anatomic and functional status of the macula. Am J Ophthalmol. 2003;136(2): 277-284.

95. Takano M, Kishi S. Foveal retinoschisis and retinal detachment in severely myopic eyes with posterior staphyloma. Am J Ophthalmol. 1999;128(4):472-476.

96. Wang S, Peng Q, Zhao P. SD-OCT use in myopic retinoschisis pre- and post-vitrectomy. Optom Vis Sci. 2012;89(5):678-683.

97. Flach AJ. The incidence, pathogenesis and treatment of cystoid macular edema following cataract surgery. Trans Am Ophthalmol Soc. 1998;96: 557-634.

98. Yonekawa Y, Kim IK. Pseudophakic cystoid macular edema. Curr Opin Ophthalmol. 2012;23(1):26-32.

99. Nikica G, Ljerka HP, Jelena P, Metez-Soldo K, Mladen B. Cystoid macular edema in anterior chamber lens implantation following posterior capsule rupture. Doc Ophthalmol. 1992;81(3):309-315.

100. Gass JDM. Stereoscopic Atlas of Macular Diseases: Diagnosis and Treatment. 4th ed. St Louis, MO: Mosby; 1997.

101. Shukla D, Gupta SR, Neelakantan N, et al. Type 2 idiopathic macular telangiectasia. Retina. 2012;32(2):265-274.

102. Hayreh SS. Posterior ciliary artery circulation in health and disease: the Weisenfeld lecture. Invest Ophthalmol Vis Sci. 2004;45(3): 749-757, 748.

103. Fujiwara T, Imamura Y, Margolis R, Slakter JS, Spaide RF. Enhanced depth imaging optical coherence tomography of the choroid in highly myopic eyes. Am J Ophthalmol. 2009;148(3):445-450.

104. Dell'Omo R, Costagliola C, Di Salvatore F, Cifariello F, Dell'Omo E. Enhanced depth imaging spectral-domain optical coherence tomography. Retina. 2010;30(2):378-379.

105. Querques G, Lattanzio R, Querques L, et al. Enhanced depth imaging optical coherence tomography in type 2 diabetes. Invest Ophthalmol Vis Sci. 2012;53(10):6017-6024. 
106. Reibaldi M, Boscia F, Avitabile T, et al. Enhanced depth imaging optical coherence tomography of the choroid in idiopathic macular hole: A crosssectional prospective study. Am J Ophthalmol. 2011;151(1): 112-117. e2.

107. Coscas F, Coscas G, Querques G, et al. En face enhanced depth imaging optical coherence tomography of fibrovascular pigment epithelium detachment. Invest Ophthalmol Vis Sci. 2012;53(7):4147-4151.
108. Arevalo JF, Maia M, Garcia-Amaris RA, et al; Pan-American Collaborative Retina Study Group. Intravitreal bevacizumab for refractory pseudophakic cystoid macular edema: the Pan-American Collaborative Retina Study Group results. Ophthalmology. 2009; 116(8):1481-1487.

\section{Publish your work in this journal}

Clinical Ophthalmology is an international, peer-reviewed journal covering all subspecialties within ophthalmology. Key topics include: Optometry; Visual science; Pharmacology and drug therapy in eye diseases; Basic Sciences; Primary and Secondary eye care; Patient Safety and Quality of Care Improvements. This journal is indexed on

\section{Dovepress}

PubMed Central and CAS, and is the official journal of The Society of Clinical Ophthalmology (SCO). The manuscript management system is completely online and includes a very quick and fair peer-review system, which is all easy to use. Visit http://www.dovepress.com/ testimonials.php to read real quotes from published authors. 